\title{
Viscosity solutions for the crystalline mean curvature flow with a nonuniform driving force term
}

\section{Yoshikazu Giga ${ }^{1} \cdot$ Norbert Požár ${ }^{2}$}

Published online: 15 October 2020

(C) The Author(s) 2020

\begin{abstract}
A general purely crystalline mean curvature flow equation with a nonuniform driving force term is considered. The unique existence of a level set flow is established when the driving force term is continuous and spatially Lipschitz uniformly in time. By introducing a suitable notion of a solution a comparison principle of continuous solutions is established for equations including the level set equations. An existence of a solution is obtained by stability and approximation by smoother problems. A necessary equi-continuity of approximate solutions is established. It should be noted that the value of crystalline curvature may depend not only on the geometry of evolving surfaces but also on the driving force if it is spatially inhomogeneous.
\end{abstract}

Mathematics Subject Classification 35K67 $\cdot 35 \mathrm{D} 40 \cdot 35 \mathrm{~K} 55 \cdot 35 \mathrm{~B} 51 \cdot 35 \mathrm{~K} 93$

\section{Introduction}

In our previous works [21, 22], we constructed a unique global-in-time level set flow for the crystalline mean curvature flow of the form

$$
V=g\left(v, \kappa_{\sigma}\right) .
$$

Here $V$ is the normal velocity of an evolving hypersurface in $\mathbb{R}^{n}, n \geq 2$, in the direction of a unit normal vector field $v$ and $\kappa_{\sigma}$ is a (purely) crystalline mean curvature of the hypersurface. The anisotropy $\sigma$ is assumed to be crystalline, that is, $\sigma: \mathbb{R}^{n} \rightarrow \mathbb{R}$ is a positively one-homogeneous function such that $\{\sigma<1\}$ is a bounded convex polytope. The function

This article is part of the topical collection Viscosity solutions-Dedicated to Hitoshi Ishii on the award of the 1st Kodaira Kunihiko Prize, edited by Kazuhiro Ishige, Shigeaki Koike, Tohru Ozawa, Senjo Shimizu.

Yoshikazu Giga

labgiga@ms.u-tokyo.ac.jp

Norbert Požár

npozar@se.kanazawa-u.ac.jp

1 Graduate School of Mathematical Sciences, University of Tokyo, 3-8-1 Komaba Meguro-ku, Tokyo 153-8914, Japan

2 Faculty of Mathematics and Physics, Institute of Science and Engineering, Kanazawa University, Kakuma town, Kanazawa, Ishikawa 920-1192, Japan 
$g \in C\left(\mathcal{S}^{n-1} \times \mathbb{R}\right)$ is a given function that is non-decreasing in the second variable so that the problem is at least formally degenerate parabolic; here, $\mathcal{S}^{n-1}$ denotes the unit sphere in $\mathbb{R}^{n}$. We are using the convention that $V=\kappa_{\sigma}$ is the usual mean curvature flow when $\sigma$ is isotropic so that $\kappa_{\sigma}$ is the usual mean curvature.

Our goal is to extend the result in [21] to the problem

$$
V=g\left(v, \kappa_{\sigma}+f(x, t)\right),
$$

where $f=f(x, t)$ is a continuous function that is Lipschitz continuous in space variable $x$ uniformly in time $t$. Namely, we consider a crystalline mean curvature flow with a nonuniform driving force term. We introduce a suitable notion of viscosity solutions to the level set equation for (1.1), which looks slightly weaker than those in [21, 22]. Our main result reads:

Theorem 1.1 Assume that $g \in C\left(\mathcal{S}^{n-1} \times \mathbb{R}\right)$ is Lipschitz continuous in the second variable uniformly in the first variable and non-decreasing in the second variable, $\sigma$ is a crystalline anisotropy and $f \in C\left(\mathbb{R}^{n} \times \mathbb{R}\right)$ is Lipschitz continuous in space uniformly in time. Then there is a unique global-in-time level set flow to (1.1) when the initial hypersurface is compact.

The assumption on $g$ prohibits superlinear growth in $\kappa_{\sigma}+f$. However, it is still quite general since it allows nonlinear dependence in $\kappa_{\sigma}+f$.

The general strategy to prove this result is along the line of [22]. However, the problem is substantially more difficult when $f$ is spatially nonuniform even if $f$ is time-independent.

We have to understand $\kappa_{\sigma}+f$ at a given time $t$ as one term given as the canonical restriction of the subdifferential of the functional

$$
\mathcal{G}_{t}(E):=\int_{\partial E} \sigma(v) d S+\int_{E} f_{t} d x,
$$

where $f_{t}:=f(\cdot, t)$, to be consistent with the formal gradient flow structure, with Chambolle et al. [8,9] and previous work in 1D in [15, 16]; see also [6, Section 2.3]. This becomes important in the proof of stability in Sect. 4.

To establish Theorem 1.1, we study the well-posedness of the level set formulation for (1.1). Following the convention of [22], we take a level set function $u=u(x, t)$ such that its every sublevel set is the solution of (1.1). Then $u$ is a solution of

$$
u_{t}+F(\nabla u, \operatorname{div}(\nabla \sigma(\nabla u))-f)=0,
$$

where

$$
F(p, \xi):=|p| g\left(\frac{p}{|p|},-\xi\right) .
$$

Let us assume that $f=f(x)$ for now to simplify the notation. To define $\kappa_{\sigma}+f$ for admissible faceted functions on their facets, we take

$$
\mathcal{E}_{f}(\psi):=\int \sigma(\nabla \psi)+f \psi d x \text { for } \psi \in \operatorname{Lip},
$$

where the integral is taken over some appropriate domain like $\mathbb{T}^{n}$, and define 


$$
\Lambda_{f}[\psi]=\operatorname{div} z_{\min }-f=-\partial^{0} \mathcal{E}_{f}(\psi),
$$

where $z_{\min }$ is a minimizer of

$$
\|\operatorname{div} z-f\|_{L^{2}}
$$

over all $z \in L^{\infty}, \operatorname{div} z \in L^{2}$ such that

$$
z \in \partial \sigma(\nabla \psi) \quad \text { a.e. }
$$

Note that $\partial \mathcal{E}_{f}(\psi)=\partial \mathcal{E}_{0}(\psi)+f$. Therefore $f$ does not change admissible functions or admissible facets, only the value of the canonical restriction $\partial^{0} \mathcal{E}_{f}(\psi)$. We will see below that $\Lambda_{f}$ satisfies a comparison principle, Proposition 2.1.

We define a notion of viscosity solutions for (1.2) (Definition 2.7), which is a generalization of the notion introduced in the previous work [21, 22] to allow for the nonuniform driving force term. We first establish a comparison principle (Theorem 3.1) for equations including the level set equation of (1.1). However, our flattening argument [14, 21, 22] requires that one of sub- and supersolutions is continuous. This requirement is unnecessary when $n=2$ since the set of singular directions of the interfacial energy $\sigma$ is compact [16]. We next prove the existence of a solution by showing the stability for relaxed limits of solutions of (1.2) with regularized $\sigma$ : both with quadratic growth (Theorem 4.1) and with linear growth (Theorem 4.4). However, to show the full convergence through the comparison principle we need to show that the relaxed semilimit must be continuous. For this purpose, we establish a uniform Lipschitz bound in space (Theorem 5.1) and a uniform 1/2-Hölder bound in time (Theorem 5.2) for an approximate solution. Although a Lipschitz bound is well known in the elliptic case even for viscosity solutions [4], it is not trivial to adapt it in the parabolic case, especially in our setting. We shall give a direct proof for a spatial Lipschitz bound for viscosity solutions for level set equations without appealing to the classical theory of quasilinear or fully nonlinear uniformly parabolic equations; see e.g. [28-30]. See, for example, also [3, 20] for applications to viscosity solutions. We also give 1/2-Hölder bound in time by constructing suitable barriers. Using these results, we deduce the unique existence of solutions of (1.2) (Theorem 5.3). The proof of Theorem 1.1 is outlined at the end of Sect. 5 .

\subsection{Literature overview}

The crystalline mean curvature flow was introduced in mathematical community by Angenent and Gurtin [1] and independently by Taylor [33] around thirty years ago. Since then, there is a large number of literature. The bibliography of [21,22] includes several key references on the crystalline mean curvature flow or flow with constant driving force term. We here mention references related to the crystalline mean curvature flow with nonuniform driving force term. The problem is far more difficult than the case of constant driving force even for the problem of planar motion because the expected speed on facets may not be constant, which may cause facet bending or splitting. A first global unique existence result has been established in [13] for a graph-like curve based on the theory of maximal monotone operators. Several explicit facet splitting solutions are constructed in [13]. In [5] planar, crystalline flow with nonuniform driving force was constructed but under the assumtion that the driving force preserves facets, in other words, facet splitting and bending does not occur. Several conditions for the preservation of facets are given especially for the Stefan problem when the anisotropy is fixed like its Wulff shape is a cylinder 
[23-25]. An explicit facet bending solution is given in a planar motion under rectangular anisotropy [17-19, 26, 27]; see also [31, 32]. For a graph-like curve a general global wellposedness results are established in $[15,16]$ for a general equation like (1.1). For a closed curve less is known. In [10] a local-in-time strong solution is constructed when the force term is Lipschitz in space-time or spatially independent for the crystalline curvature flow in the plane. It is quite recent that the level set approach is extended to higher dimensional setting even for constant driving force [21, 22]. In [8], by extending the method in [9] for $V=\sigma(v) \kappa_{\sigma}$, quite general driving force is allowed but the equation is of the form $V=$ $M(v)\left(\kappa_{\sigma}+f\right)$ with "convex" mobility. They proved the unique existence of the level set flow for any initial hypersurface which may be unbounded. In our notation, they assume that $g$ is linear in the last variable. Although they allow general anisotropy $\sigma$, not necessarily purely crystalline, their method essentially depends on linearity of $g$ in the last variable. Their method is substantially different from ours.

This paper is organized as follows. In Sect. 2, we introduce a notion of a viscosity solution. In Sect. 3, a comparison principle is established. In Sect. 4, we prove stability of a solution. In Sect. 5, we prove a necessary Lipschitz bound in space and 1/2-Hölder bound in time to show the continuity of the limit. In Sect. 6, we warn that our value $\kappa_{\sigma}+f$ is not a simple sum of $\kappa_{\sigma}$ and $f$. In the "Appendix A", we give a proof of a Lipschitz bound of our resolvent problem.

\section{Viscosity solutions}

\subsection{Facet}

Let $\sigma$ be an anisotropy, that is, let $\sigma: \mathbb{R}^{n} \rightarrow \mathbb{R}$ be positively one-homogeneous function such that $\{\sigma<1\}$ is a bounded convex set. Suppose that $U \subset \mathbb{R}^{n}$ is an open set and $\psi \in \operatorname{Lip}(U)$. We define the set of Cahn-Hoffman vector fields for $\psi$ as

$$
C H(\psi ; U):=\left\{z \in X^{2}(U): z \in \partial \sigma(\nabla \psi) \text { a. e. }\right\} .
$$

Here $X^{2}(U):=\left\{z \in L^{\infty}(U): \operatorname{div} z \in L^{2}(U)\right\}$; see [2]. If $C H(\psi ; U)$ is nonempty, we define the $\sigma^{\circ}-\left(L^{2}\right)$ divergence of $\psi$ for any $f \in L^{2}(U)$ as

$$
\Lambda_{f}[\psi]:=\operatorname{div} z_{\min }-f \quad \text { on }\{\psi=0\},
$$

where $z_{\min } \in C H(\psi ; U)$ minimizes $z \mapsto\|\operatorname{div} z-f\|_{L^{2}(U)}$. Note that since

$$
\operatorname{div} C H(\psi ; U):=\{\operatorname{div} z: z \in C H(\psi ; U)\}
$$

is a closed, convex and nonempty (by assumption) subset of $L^{2}(U)$ there exists a unique minimizing div $z_{\min }$ (but $z_{\min }$ might not be unique).

Let us recall the comparison principle for $\sigma^{\circ}-\left(L^{2}\right)$ minimal divergence, proved in [21] for $f \equiv 0$. The generalization to $f \not \equiv 0$ is straightforward.

Proposition 2.1 (cf. [21, Proposition 4.12]) If $\psi_{1}, \psi_{2}$ are two Lipschitz functions on an open set $U$ such that their zero sets are compact subsets of $U \subset \mathbb{R}^{n}, f_{1}, f_{2} \in L^{2}(U)$ and $\Lambda_{f_{i}}\left[\psi_{i}\right], i=1,2$, are well-defined, then

$$
\operatorname{sign} \psi_{1} \leq \operatorname{sign} \psi_{2} \quad \text { on } U \text { and } f_{1} \geq f_{2} \quad \text { a. e. on } U
$$


imply

$$
\Lambda_{f_{1}}\left[\psi_{1}\right] \leq \Lambda_{f_{2}}\left[\psi_{2}\right], \quad \text { a. e. on }\left\{\psi_{1}=0\right\} \cap\left\{\psi_{2}=0\right\}
$$

The dependence of the minimal divergence only on sign $\psi$ motivates the following definition. Let $\mathcal{F}=\left\{\xi \mid \xi: \mathbb{R}^{n} \rightarrow \mathbb{R}\right\}$ be the set of all real-valued functions on $\mathbb{R}^{n}$. We define the relation on $\mathcal{F}$ as

$$
\xi_{1} \sim \xi_{2} \Leftrightarrow \operatorname{sign} \xi_{1}=\operatorname{sign} \xi_{2}
$$

where sign $s=+1,0,-1$, respectively, when $s>0, s=0, s<0$. The relation $\sim$ defines an equivalence relation on $\mathcal{F}$. We refer to the equivalence classes $[\xi]:=\{\psi: \psi \sim \xi\}$ of $\mathcal{F}$ with respect to $\sim$ as (abstract) facets. We write $\left[\xi_{1}\right] \preceq\left[\xi_{2}\right]$ when sign $\xi_{1} \leq \operatorname{sign} \xi_{2}$ and this relation defines a total order on the set of all facets $\mathcal{F} / \sim:=\{[\xi]: \xi \in \mathcal{F}\}$.

We say that a facet $[\xi]$ is a $\sigma^{\circ}-\left(L^{2}\right)$ Cahn-Hoffman facet if $\{\xi=0\}$ is compact and there are an open set $U \subset \mathbb{R}^{n},\{\xi=0\} \subset U$, and a Lipschitz function $\psi \in[\xi]$ such that $C H(\psi ; U) \neq \emptyset$.

Remark 2.2 The definition of $\sigma^{\circ}-\left(L^{2}\right)$ Cahn-Hoffman facet guarantees that there exists a periodic Lipschitz function $\zeta$ on $\mathbb{R}^{n}$ and a periodic vector field $z \in L^{\infty}\left(\mathbb{R}^{n}\right)$ such that $\zeta=\psi$ in a neighborhood of $\{\xi=0\}, z \in \partial \sigma(\nabla \zeta)$ a. e. and $\operatorname{div} z \in L_{\text {loc }}^{2}\left(\mathbb{R}^{n}\right)$. Indeed, we can assume that $U$ is bounded and hence $\partial U$ is compact. We set $\varepsilon:=\min _{\partial U}|\psi| / 2>0$ and consider $\theta:=\min (\varepsilon, \max (-\varepsilon, \psi))$. Clearly $z \in \partial \sigma(\nabla \theta)$ a. e. as $\partial \sigma(p) \subset \partial \sigma(0)$ for any $p \in \mathbb{R}^{n}$. When $n \geq 2$, either $\theta \equiv \varepsilon$ or $\theta \equiv-\varepsilon$ outside of a large ball and hence a periodic extension is trivial. When $n=1$ we might have to modify $\theta$ by an even extension with respect to some point outside of $U$ to guarantee that $\theta$ has one sign outside of a large ball. For details see [21]. This allows us to consider facets as objects on $\mathbb{T}^{n}$.

For a $\sigma^{\circ}-\left(L^{2}\right)$ Cahn-Hoffman facet $[\xi]$ and $f \in L^{2}\left(\mathbb{R}^{n}\right)$ we define the $\sigma^{\circ}-\left(L^{2}\right)$ minimal divergence of the facet $[\xi], \Lambda[\xi] \in L^{2}(\{\xi=0\})$, as

$$
\Lambda_{f}[\xi]:=\operatorname{div} z_{\min }-f \quad \text { on }\{\xi=0\},
$$

where $z_{\min } \in C H(\psi ; U)$ minimizes $\quad z \mapsto\|\operatorname{div} z-f\|_{L^{2}(U)}$ for some $U \subset \mathbb{R}^{n}$ open, $\{\xi=0\} \subset U$, and Lipschitz $\psi \in[\xi]$ such that $C H(\psi ; U) \neq \emptyset$. Note that $\Lambda_{f}[\xi]$ is welldefined since it does not depend on the choice of $\psi$ or $U$ by Proposition 2.1, and the notation is consistent with (2.1) since $\psi \in[\psi]$.

If $f$ is locally bounded, by comparison with Wulff functions like $\max \left(\sigma^{\circ}-c, 0\right)$ we can show that $\Lambda_{f}[\xi]$ is locally bounded on the interior of $\{\xi=0\}$ by Proposition 2.1 and we can define

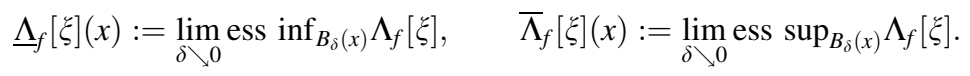

These values might differ since $\Lambda_{f}[\xi]$ is in general discontinuous. 
Lemma 2.3 For any $\sigma^{\circ}-\left(L^{2}\right)$ Cahn-Hoffman facet $[\chi]$ and $f \in L^{2}$,

$$
\Lambda_{f+c}[\chi]=\Lambda_{f}[\chi]-c \quad \text { a.e. on }\{\chi=0\} \text { for any constant } c \in \mathbb{R} \text {. }
$$

Proof We have $\Lambda_{f}[\chi]=\operatorname{div} z_{\min }-f$ on $\{\chi=0\}$ where $z_{\min }$ minimizes $z \mapsto\|\operatorname{div} z-f\|_{L^{2}(U)}$ over $z \in C H(\psi ; U)$ for some open $U \supset\{\chi=0\}$ and Lipschitz $\psi \in[\chi]$. By Remark 2.2 we can assume that $\psi \in \operatorname{Lip}\left(\mathbb{T}^{n}\right), \chi \in L^{2}\left(\mathbb{T}^{n}\right)$. To simplify the notation, let us set $C:=$ $\left\{\operatorname{div} z: z \in C H\left(\psi ; \mathbb{T}^{n}\right)\right\}$ and let $P_{E} x$ denote the element of $E$ closest to $x$ for $E \subset L^{2}\left(\mathbb{T}^{n}\right)$ closed, convex and $x \in L^{2}\left(\mathbb{T}^{n}\right)$. We have $\Lambda_{f}[\chi]=P_{C-f} 0=P_{C} f-f$ on $\{\chi=0\}$.

To deduce (2.4), we only need to show that $P_{C} f=P_{C}(f+c)$ for all $c \in \mathbb{R}$. Observe that $C \subset 1^{\perp}$ where $1^{\perp} \subset L^{2}\left(\mathbb{T}^{n}\right)$ is the orthogonal subspace to $1 \in L^{2}\left(\mathbb{T}^{n}\right)$. Indeed, by Green's theorem [2]

$$
(1, \xi)_{L^{2}\left(\mathbb{T}^{n}\right)}=(1, \operatorname{div} z)_{L^{2}\left(\mathbb{T}^{n}\right)}=0 \quad \text { for all } \xi=\operatorname{div} z \in C .
$$

In particular, the Pythagorean theorem implies

$$
P_{C}(f+c)=P_{C} P_{1^{\perp}}(f+c)=P_{C} P_{1^{\perp}} f=P_{C} f .
$$

(2.4) follows.

Let us emphasize that Lemma 2.3 holds only for constant $c$. It is not in general true that $\Lambda_{f}=\Lambda_{0}-f$.

Corollary 2.4 If $\left|f_{1}-f_{2}\right| \leq M$ then

$$
\left|\Lambda_{f_{1}}[\chi]-\Lambda_{f_{2}}[\chi]\right| \leq M \quad \text { a.e. on }\{\chi=0\} .
$$

Proof We have $f_{1} \leq f_{2}+M$ and so by the comparison principle Proposition 2.1 and the dependence of $\Lambda_{f}$ on shifts of $f$ by constants in Lemma 2.3, we have

$$
\Lambda_{f_{1}}[\chi] \geq \Lambda_{f_{2}+M}[\chi]=\Lambda_{f_{2}}[\chi]-M .
$$

The other inequality is analogous.

Although in this paper we do not use the next Corollary on the stability of $\Lambda$ with respect to $f$, we give it as a simple application of Corollary 2.4.

Corollary 2.5 If $f_{k} \rightrightarrows f$ then

$$
\left\|\Lambda_{f_{k}}[\chi]-\Lambda_{f}[\chi]\right\|_{L^{\infty}(\{\chi=0\})} \rightarrow 0 \quad \text { as } k \rightarrow \infty .
$$

Proof Clear from Corollary 2.4. 


\subsection{Slicing}

From this point on we shall assume that $\sigma$ is a crystalline anisotropy, that is, the set $\{\sigma<1\}$ is a polytope. Based on a fixed gradient $\hat{p} \in \mathbb{R}^{n}$, we consider the orthogonal decomposition of the space $\mathbb{R}^{n}=Z \oplus Z^{\perp}$ so that $Z$ is the linear subspace parallel to the affine hull aff $\partial \sigma(\hat{p})$. In other words, $Z$ is the smallest subspace such that $\partial \sigma(\hat{p}) \subset Z+\xi$ for some $\xi \in \mathbb{R}^{n}$. Let $k:=\operatorname{dim} \partial \sigma(\hat{p}):=\operatorname{dim} Z$. By fixing orthogonal bases of $Z$ and $Z^{\perp}$, we can fix two linear isometries $\mathcal{T}: \mathbb{R}^{k} \rightarrow Z$ and $\mathcal{T}_{\perp}: \mathbb{R}^{n-k} \rightarrow Z^{\perp}$. Then we can write every $x \in \mathbb{R}^{n}$ uniquely as $x=\mathcal{T} x^{\prime}+\mathcal{T}_{\perp} x^{\prime \prime}$, where $x^{\prime} \in \mathbb{R}^{k}$ and $x^{\prime \prime} \in \mathbb{R}^{n-k}$. For $k=0$ or $k=n$, we take $x=x^{\prime \prime}$ or $x=x^{\prime}$, respectively. Note that $x^{\prime}=\mathcal{T}^{*} x$ and $x^{\prime \prime}=\mathcal{T}_{\perp}^{*} x$, where $\mathcal{T}^{*}$ and $\mathcal{T}_{\perp}^{*}$ are the adjoints of $\mathcal{T}$ and $\mathcal{T}_{\perp}$, respectively. The precise choice of $\mathcal{T}$ and $\mathcal{T}_{\perp}$ for each $\hat{p} \in \mathbb{R}^{n}$ is not important as long as we use it consistently.

Using this decomposition, we introduce the positively one-homogeneous function $\sigma_{\hat{p}}^{\text {sl }}$ : $\mathbb{R}^{k} \rightarrow \mathbb{R}$ as

$$
\sigma_{\hat{p}}^{\mathrm{sl}}(w):=\lim _{\lambda \rightarrow 0+} \frac{\sigma(\hat{p}+\lambda \mathcal{T} w)-\sigma(\hat{p})}{\lambda}, \quad w \in \mathbb{R}^{k}
$$

This function represents the infinitesimal structure of $\sigma$ near $\hat{p}$, sliced in the direction of $Z$.

For a $\left(\sigma_{\hat{p}}^{\mathrm{sl}}\right)^{\circ}-\left(L^{2}\right)$ Cahn-Hoffman facet $[\chi]$ on $\mathbb{R}^{k}$ and $f \in L^{2}\left(\mathbb{R}^{k}\right)$, we denote $\Lambda_{f}[\chi]$ as defined in (2.2) with $\sigma=\sigma_{\hat{p}}^{\text {sl }}$ on $\mathbb{R}^{k}$ by $\Lambda_{\hat{p}, f}[\chi]$.

With $\hat{p}, k$ as above, we say that a function $\psi \in \operatorname{Lip}\left(\mathbb{R}^{k}\right)$ is a $\hat{p}$-admissible support function if $[\psi]$ is a $\left(\sigma_{\hat{p}}^{\mathrm{sl}}\right)^{\circ}-\left(L^{2}\right)$ Cahn-Hoffman facet.

\subsection{Definition of viscosity solutions}

We generalize the definition from [22]. We recall the definition of test functions. The variables $x^{\prime}=\mathcal{T}^{*} x, x^{\prime \prime}=\mathcal{T}_{\perp}^{*} x$ refer to the sliced decomposition introduced in Sect. 2.2.

Definition 2.6 (cf. [22, Definition 4.7]) Let $\hat{p} \in \mathbb{R}^{n},(\hat{x}, \hat{t}) \in \mathbb{R}^{n} \times \mathbb{R}, k:=\operatorname{dim} \partial \sigma(\hat{p})$. We say that $\varphi(x, t)=\psi\left(x^{\prime}\right)+\theta\left(x^{\prime \prime}\right)+\hat{p} \cdot x+g(t)$ is an admissible stratified faceted test function at $(\hat{x}, \hat{t})$ with slope $\hat{p}$ if $\theta \in C^{1}\left(\mathbb{R}^{n-k}\right), \nabla \theta\left(\hat{x}^{\prime \prime}\right)=0, g \in C^{1}(\mathbb{R})$, and $\psi \in \operatorname{Lip}\left(\mathbb{R}^{k}\right)$ is a $\hat{p}$-admissible support function with $\hat{x}^{\prime} \in \operatorname{int}\{\psi=0\}$. Note that if $k=0$, we have $\varphi(x, t)=\theta(x)+g(t)$ for some $\theta \in C^{1}\left(\mathbb{R}^{n}\right), g \in C^{1}(\mathbb{R})$.

Definition 2.7 (Viscosity solution, $c f$. [21, Definition 5.2]) We say that an upper semicontinuous function $u$ is a viscosity subsolution of

$$
u_{t}+F(x, t, \nabla u, \operatorname{div}(\nabla \sigma(\nabla u))-f)=0
$$

on $\mathbb{R}^{n} \times(0, T), T>0$, if for any $\hat{p} \in \mathbb{R}^{n}, \hat{x} \in \mathbb{R}^{n}, \hat{t} \in(0, T)$ and any admissible stratified faceted test function $\varphi$ at $(\hat{x}, \hat{t})$ with slope $\hat{p}$ of the form $\varphi(x, t)=\psi\left(x^{\prime}\right)+\theta\left(x^{\prime \prime}\right)+\hat{p} \cdot x+$ $g(t)$ such that the function $u-\varphi(\cdot-h)$ has a global maximum on $\mathbb{R}^{n} \times(0, T)$ at $(\hat{x}, \hat{t})$ for all sufficiently small $h^{\prime} \in \mathbb{R}^{k}$ and $h^{\prime \prime}=0$, then

$$
g^{\prime}(\hat{t})+F\left(\hat{x}, \hat{t}, \hat{p}, \underline{\Lambda}_{\hat{p}, \hat{f}}[\psi]\left(\hat{x}^{\prime}\right)\right) \leq 0,
$$

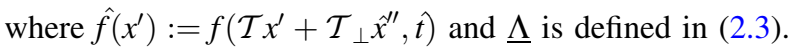


Viscosity supersolutions are defined analogously as lower semicontinuous functions, replacing a global maximum with a global minimum, $\underline{\Lambda}$ with $\bar{\Lambda}$, and reversing the inequality in (2.7).

A continuous function that is both a viscosity subsolution and a viscosity supersolution is called a viscosity solution.

Note that (2.7) is weaker than the condition in [21, 22] since

$$
\text { ess } \inf _{B_{\delta}(x)} \Lambda_{\hat{p}, \hat{f}}[\psi] \leq \Lambda_{\hat{p}, \hat{f}}[\psi](x) \text { for any } \delta>0 \text {. }
$$

This is to allow for the dependence of $f$ on $x$ and it will become important in the proof of stability in Sect. 4 since the right-hand side of (4.5) is not quite zero. But this relaxed condition (2.7) is still strong enough for the comparison principle to hold.

\section{Comparison principle}

We will establish the comparison principle between a viscosity subsolution $u$ and a viscosity supersolution $v$ under the additional assumption that at least one of them is continuous. This is enough to show the existence of solutions by approximation as we can obtain a uniform modulus of continuity for the approximating solutions; see Sect. 5. At this time we do not know how to establish the comparison principle for semicontinuous solutions.

In this section we allow for an explicit dependence of $F$ on the variables $x$ and $t$. If we do not assume further regularity on at least one of the solutions, we will need to also assume that $F$ satisfies

$$
|F(x, t, p, \xi)-F(y, s, p, \eta)| \leq C(|p|+1)(|x-y|+|t-s|+|\xi-\eta|)
$$

for some constant $C$. This covers both crystalline mean curvature flows with

$$
F(p, \xi)=-\beta(p) \xi
$$

and anisotropic total variation flows

$$
F(p, \xi)=-\xi
$$

but forbids superlinear growth of $F$ in the last variable.

Theorem 3.1 (Comparison principle) Let $\sigma$ be a crystalline anisotropy, $F \in C\left(\mathbb{R}^{n} \times \mathbb{R} \times\right.$ $\left.\mathbb{R}^{n} \times \mathbb{R}\right)$ be non-increasing in the last variable, and $f \in C\left(\mathbb{R}^{n} \times \mathbb{R}\right)$ be Lipschitz continuous in space, uniformly in time. Let $u$ be a viscosity subsolution of (2.6) and let $v$ be a continuous viscosity supersolution of (2.6) on $Q_{T}:=\mathbb{R}^{n} \times(0, T)$ for some $T>0$ in the sense of Definition 2.7. Suppose that $u$ and $v$ are bounded and that there exist constants $R>0, a \leq b$ such that $u \equiv a$ and $v \equiv b$ on $\left(\mathbb{R}^{n} \backslash B_{R}(0)\right) \times(0, T)$. Suppose that either

(i) $\quad v$ is Lipschitz continuous in space, uniformly in time; or

(ii) F satisfies (3.1) and $f$ is Lipschitz continuous in both variables.

Then

$$
u(\cdot, 0) \leq v(\cdot, 0) \quad \text { on } \mathbb{R}^{n}
$$


implies

$$
u \leq v \quad \text { on } Q_{T}
$$

We point out that only the space continuity of $v$ is necessary for the conclusion of the theorem. It allows us to pass in the limit in the last step of the proof of the Proposition 3.2, and this lets us control the size of the space gradient of test functions.

Proof Suppose that $\sup _{Q_{T}}(u-v)>0$. Let us fix $\mu>0$ such that

$$
\sup _{Q_{T}} w=: m_{0}>0 .
$$

where

$$
w(x, t)=u(x, t)-v(x, t)-\frac{\mu}{T-t} .
$$

Let $(\hat{x}, \hat{t}) \in Q_{T}$ be such that $w(\hat{x}, \hat{t})=m_{0}$.

Consider

$$
\Psi_{\zeta, \varepsilon}(x, t, y, s):=u(x, t)-v(y, s)-\frac{|x-y-\zeta|^{2}}{2 \varepsilon}-\frac{|t-s|^{2}}{2 \varepsilon}-\frac{\mu}{T-t} .
$$

For $\varepsilon>0$ small enough and $|\zeta| \leq \sqrt{m_{0} \varepsilon}$ this function has a maximum on $Q_{T} \times Q_{T}$.

Proposition 3.2 Assume that $u$ and $v$ satisfy all the assumptions in Theorem 3.1. Let $\left(x_{\varepsilon}, t_{\varepsilon}, y_{\varepsilon}, s_{\varepsilon}\right)$ be a sequence of maxima of $\Psi_{\zeta_{\varepsilon}, \varepsilon}$ for some sequence $\left|\zeta_{\varepsilon}\right| \leq \sqrt{m_{0} \varepsilon}$. Then there is a constant $M$, independent of $\varepsilon$, so that we have

$$
\left|x_{\varepsilon}-y_{\varepsilon}-\zeta_{\varepsilon}\right| \leq \sqrt{M \varepsilon}, \quad\left|t_{\varepsilon}-s_{\varepsilon}\right| \leq \sqrt{M \varepsilon},
$$

and

$$
\frac{\left|x_{\varepsilon}-y_{\varepsilon}-\zeta_{\varepsilon}\right|^{2}}{\varepsilon} \rightarrow 0 \quad \text { as } \varepsilon \rightarrow 0
$$

Note that if $\zeta_{\varepsilon}=0$ the limit (3.4) is standard in the viscosity theory; see for example [7, Proposition 3.7]. However, the standard proof does not seem to apply with $\zeta_{\varepsilon} \neq 0$ and we need a continuity of $u$ or $v$ to recover (3.4). The proof of Proposition 3.2 uses the idea in [11]. We need (3.4) to show that the left-hand side of (3.8) below converges to zero as $\varepsilon \rightarrow 0$.

Proof By maximality

$$
\Psi_{\zeta_{\varepsilon}, \varepsilon}\left(x_{\varepsilon}, t_{\varepsilon}, y_{\varepsilon}, s_{\varepsilon}\right) \geq \Psi_{\zeta_{\varepsilon}, \varepsilon}(\hat{x}, \hat{t}, \hat{x}, \hat{t})=w(\hat{x}, \hat{t})-\frac{\left|\zeta_{\varepsilon}\right|^{2}}{2 \varepsilon} \geq \frac{m_{0}}{2}>0 .
$$

We deduce (3.3) as $u(x, t)-v(y, s)$ is bounded above by some constant $M$ on $Q_{T} \times Q_{T}$.

Since at least one of $\left(x_{\varepsilon}, t_{\varepsilon}\right)$ and $\left(y_{\varepsilon}, s_{\varepsilon}\right)$ must lie in $B_{R}(0) \times[0, T)$, we can assume that 
$\left(x_{\varepsilon}, t_{\varepsilon}, y_{\varepsilon}, s_{\varepsilon}\right) \rightarrow(\bar{x}, \bar{t}) \in \overline{B_{R}(0)} \times(0, T)$ along a subsequence. Indeed, $\bar{t}>0$ by (3.2) and $\bar{t}<T$ since $\sup \Psi_{\zeta_{\varepsilon}, \varepsilon}(\cdot, t, \cdot, \cdot) \rightarrow-\infty$ as $t \rightarrow T-$.

Therefore we have

$$
\begin{aligned}
& u(\bar{x}, \bar{t})-v\left(\bar{x}-\zeta_{\varepsilon}, \bar{t}\right)-\mu(T-\vec{t})^{-1}=\Psi_{\zeta_{\varepsilon}, \varepsilon}\left(\bar{x}, \bar{t}, \bar{x}-\zeta_{\varepsilon}, \vec{t}\right) \\
& \quad \leq \Psi_{\zeta_{\varepsilon}, \varepsilon}\left(x_{\varepsilon}, t_{\varepsilon}, y_{\varepsilon}, s_{\varepsilon}\right) \\
& \quad=u\left(x_{\varepsilon}, t_{\varepsilon}\right)-v\left(y_{\varepsilon}, s_{\varepsilon}\right)-\frac{\left|x_{\varepsilon}-y_{\varepsilon}-\zeta_{\varepsilon}\right|^{2}}{2 \varepsilon}-\frac{\left|t_{\varepsilon}-s_{\varepsilon}\right|^{2}}{2 \varepsilon}-\mu\left(T-t_{\varepsilon}\right)^{-1} .
\end{aligned}
$$

After rearranging,

$$
\begin{gathered}
\frac{\left|x_{\varepsilon}-y_{\varepsilon}-\zeta_{\varepsilon}\right|^{2}}{2 \varepsilon}+\frac{\left|t_{\varepsilon}-s_{\varepsilon}\right|^{2}}{2 \varepsilon} \leq \\
u\left(x_{\varepsilon}, t_{\varepsilon}\right)-u(\bar{x}, \bar{t})+v\left(\bar{x}-\zeta_{\varepsilon}, \bar{t}\right)-v\left(y_{\varepsilon}, s_{\varepsilon}\right) \\
+\mu(T-\bar{t})^{-1}-\mu\left(T-t_{\varepsilon}\right)^{-1} .
\end{gathered}
$$

Taking lim $\sup _{\varepsilon \rightarrow 0+}$ of both sides, and using the upper semicontinuity of $u$ and the continuity of $v$, we recover (3.4). Since every subsequence has a subsequence with limit 0 , we conclude that (3.4) holds for the full sequence.

We showed in [21, Prop. 7.4 ] the following.

Lemma 3.3 There exists $\varepsilon_{0}>0$ such that for every fixed $0<\varepsilon<\varepsilon_{0}$ there is a set $\Xi_{\varepsilon} \subset \mathbb{R}^{n}$ on which $\partial \sigma$ is constant, and $\zeta_{\varepsilon} \in \mathbb{R}^{n}, \lambda_{\varepsilon}>0$ with $\left|\zeta_{\varepsilon}\right|+2 \lambda_{\varepsilon}<\kappa(\varepsilon):=\sqrt{m_{0} \varepsilon}$ such that for every $\zeta,\left|\zeta-\zeta_{\varepsilon}\right| \leq 2 \lambda_{\varepsilon}$ there is a maximizer $(x, t, y, s)$ of $\Psi_{\zeta_{\varepsilon}, \varepsilon}$ on $Q_{T} \times Q_{T}$ such that

$$
\frac{x-y-\zeta}{\varepsilon} \in \Xi_{\varepsilon} .
$$

Due to a bit of convex analysis explained in [21], $\Xi_{\varepsilon}-\Xi_{\varepsilon} \subset(\text { aff } \partial \sigma(p))^{\perp}$ for every $p \in \Xi_{\varepsilon}$ and therefore aff $\partial \sigma(p) \subset\left(\Xi_{\varepsilon}-\Xi_{\varepsilon}\right)^{\perp}$.

We set $Z_{\varepsilon}:=\operatorname{aff} \partial \sigma(p)$ for some $p \in \Xi_{\varepsilon}, k_{\varepsilon}:=\operatorname{dim} Z_{\varepsilon}$, with the linear isometries $\mathcal{T}_{\varepsilon}$ : $\mathbb{R}^{k} \rightarrow Z_{\varepsilon}$ and $\mathcal{T}_{\perp, \varepsilon}: \mathbb{R}^{n-k} \rightarrow Z_{\varepsilon}^{\perp}$. Since $p \perp Z_{\varepsilon}$ because $\sigma$ is one-homogeneous, the consequence of the flatness lemma, [21, Lemma 7.6], reduces to:

Lemma 3.4 For $\zeta_{\varepsilon}, \lambda_{\varepsilon}$ and $Z_{\varepsilon}$ as above, we have

$$
\ell_{\varepsilon}(\zeta)=\text { const } \quad \text { for } \zeta \in \zeta_{\varepsilon}+Z_{\varepsilon},\left|\zeta-\zeta_{\varepsilon}\right| \leq 2 \lambda_{\varepsilon},
$$

where

$$
\ell_{\varepsilon}(\zeta):=\sup _{Q_{T} \times Q_{T}} \Psi_{\zeta, \varepsilon}
$$

Choosing some point of maximum $\left(x_{\varepsilon}, t_{\varepsilon}, y_{\varepsilon}, s_{\varepsilon}\right) \in Q_{T} \times Q_{T}$ of $\Psi_{\zeta_{\varepsilon}, \varepsilon}$ and setting $p_{\varepsilon}:=\left(x_{\varepsilon}-y_{\varepsilon}-\zeta_{\varepsilon}\right) / \varepsilon$, we can follow the construction of $p_{\varepsilon}$-admissible faceted test functions in $[21,22]$. This gives us two admissible stratified faceted test functions $\varphi_{u}, \varphi_{v}$ for $u$ 
and $v$ at points $\left(x_{\varepsilon}, t_{\varepsilon}\right)$ and $\left(y_{\varepsilon}, s_{\varepsilon}\right)$, respectively, with $p_{\varepsilon}$-admissible support functions $\psi_{u}, \psi_{v}$ (see (3.5) below) that satisfy

$$
\operatorname{sign} \psi_{u}\left(\cdot+x_{\varepsilon}^{\prime}\right) \leq \operatorname{sign} \psi_{v}\left(\cdot+y_{\varepsilon}^{\prime}\right),
$$

and there is $\delta_{\varepsilon}>0$ with $B_{\delta_{\varepsilon}}(0) \subset\left\{\psi_{u}\left(\cdot+x_{\varepsilon}^{\prime}\right)=0\right\} \cap\left\{\psi_{v}\left(\cdot+y_{\varepsilon}^{\prime}\right)=0\right\}$. Here $x_{\varepsilon}^{\prime}:=\mathcal{T}_{\varepsilon}^{*} x_{\varepsilon}$ and so on as before. Note that $\varphi_{u}, \varphi_{v}$ and $\psi_{u}, \psi_{v}$ depend on $\varepsilon$.

Let us briefly recall how the test functions are constructed; for more details and a justification of the construction we refer the reader to [22]. We define

$$
u_{\varepsilon}(w):=u\left(\mathcal{T}_{\varepsilon} w+x_{\varepsilon}, t_{\varepsilon}\right), \quad v_{\varepsilon}(w):=v\left(\mathcal{T}_{\varepsilon} w+y_{\varepsilon}, s_{\varepsilon}\right),
$$

and

$$
U_{\varepsilon}:=\left\{u_{\varepsilon} \geq 0\right\}, \quad V_{\varepsilon}:=\left\{v_{\varepsilon} \leq 0\right\} .
$$

We set $r_{\varepsilon}:=\lambda_{\varepsilon} / 5$ and define the facet

$$
\chi_{u}(w):=\left\{\begin{array}{l}
1, \quad \operatorname{dist}\left(w, U_{\varepsilon}\right)<2 r_{\varepsilon} \text { and } \operatorname{dist}\left(w, V_{\varepsilon}\right)>\lambda_{\varepsilon}-r_{\varepsilon} \\
-1, \quad \operatorname{dist}\left(w, U_{\varepsilon}\right)>2 r_{\varepsilon} \\
0, \quad \text { otherwise. }
\end{array}\right.
$$

By the facet approximation result in [22, Th. 1.3] with $\rho=r_{\varepsilon}$ and anisotropy $\sigma_{p_{\varepsilon}}^{\text {sl }}$, there exists a $\left(\sigma_{p_{\varepsilon}}^{\mathrm{sl}}\right)^{\circ}-\left(L^{2}\right)$ Cahn-Hoffman facet $\left[\tilde{\chi}_{u}\right]$ with $\left[\chi_{u}\right] \preceq\left[\tilde{\chi}_{u}\right] \preceq\left[\sup _{\bar{B}_{r_{\varepsilon}}(\cdot)} \chi_{u}\right]$. In other words, the Cahn-Hoffman facet approximates the original facet within $r_{\varepsilon}$ in Hausdorff distance. We then set

$$
\psi_{u}\left(w+x_{\varepsilon}^{\prime}\right):=\max \left(M \operatorname{dist}\left(w,\left\{\tilde{\chi}_{u}=0\right\}\right) \operatorname{sign} \tilde{\chi}_{u}(w), m\right),
$$

where $m<0$ and $M>0$ are constants chosen so that $u_{\varepsilon} \leq \psi_{u}$. The test function $\varphi_{u}$ can be then taken as

$$
\varphi_{u}(x, t):=\psi_{u}\left(x^{\prime}\right)+\frac{\left|x^{\prime \prime}-y_{\varepsilon}^{\prime \prime}-\zeta_{\varepsilon}^{\prime \prime}\right|^{2}}{2 \varepsilon}+\frac{\left|t-s_{\varepsilon}\right|^{2}}{2 \varepsilon}+\frac{\mu}{T-t},
$$

with an appropriate modification for $\left(x^{\prime \prime}, t\right)$ away from a neighborhood of $\left(x_{\varepsilon}^{\prime \prime}, t_{\varepsilon}\right)$ if necessary to ensure that $u-\varphi_{u}$ has a global maximum at $\left(x_{\varepsilon}, t_{\varepsilon}\right)$. It is easy to check that $\varphi_{u}$ is an admissible stratified faceted test function at $\left(x_{\varepsilon}, t_{\varepsilon}\right)$ with slope $p_{\varepsilon} . \varphi_{v}$ and $\psi_{v}$ are constructed similarly.

Then Proposition 2.1 followed by Corollary 2.4 yield

$$
\begin{aligned}
& \operatorname{ess} \inf \left[\Lambda_{p_{\varepsilon}, f_{u}\left(\cdot+x_{\varepsilon}^{\prime}\right)}\left[\psi_{u}\left(\cdot+x_{\varepsilon}^{\prime}\right)\right]\right] \leq \operatorname{ess} \sup \left[\Lambda_{p_{\varepsilon} f_{u}\left(\cdot+x_{\varepsilon}^{\prime}\right)}\left[\psi_{v}\left(\cdot+y_{\varepsilon}^{\prime}\right)\right]\right] \\
& \quad \leq \operatorname{ess} \sup \left[\Lambda_{p_{\varepsilon}, f_{v}\left(\cdot+y_{\varepsilon}^{\prime}\right)}\left[\psi_{v}\left(\cdot+y_{\varepsilon}^{\prime}\right)\right]\right]+L_{f}\left|x_{\varepsilon}-y_{\varepsilon}\right|+\omega_{f}\left(\left|t_{\varepsilon}-s_{\varepsilon}\right|\right),
\end{aligned}
$$

where $f_{u}(w):=f\left(\mathcal{T} w+\mathcal{T}_{\perp} x_{\varepsilon}^{\prime \prime}, t_{\varepsilon}\right), f_{v}(w):=f\left(\mathcal{T} w+\mathcal{T}_{\perp} y_{\varepsilon}^{\prime \prime}, s_{\varepsilon}\right)$ and $L_{f}$ is the Lipschitz constant of $f$ in space and $\omega_{f}$ is the modulus of continuity of $f$ on a sufficiently large bounded set, and ess inf and ess sup are taken over $B_{\delta}(0)$. Since $\delta$ can be taken arbitrarily small, we deduce

$$
\underline{\Lambda}_{p_{\varepsilon}, f_{u}}\left[\psi_{u}\right]\left(x_{\varepsilon}^{\prime}\right) \leq \bar{\Lambda}_{p_{\varepsilon}, f_{v}}\left[\psi_{v}\right]\left(y_{\varepsilon}^{\prime}\right)+L_{f}\left|x_{\varepsilon}-y_{\varepsilon}\right|+\omega_{f}\left(\left|t_{\varepsilon}-s_{\varepsilon}\right|\right)
$$

Then we have from the definition of viscosity subsolution and supersolution 


$$
\begin{gathered}
\frac{\mu}{\left(T-t_{\varepsilon}\right)^{2}}+F\left(x_{\varepsilon}, t_{\varepsilon}, p_{\varepsilon}, \underline{\Lambda}_{p_{\varepsilon} f_{u}}\left[\psi_{u}\right]\left(x_{\varepsilon}^{\prime}\right)\right) \\
-F\left(y_{\varepsilon}, s_{\varepsilon}, p_{\varepsilon}, \bar{\Lambda}_{p_{\varepsilon} f_{v}}\left[\psi_{v}\right]\left(y_{\varepsilon}^{\prime}\right)\right) \leq 0 .
\end{gathered}
$$

On the other hand, using (3.6) and the monotonicity of $F$ in the last variable, we can estimate

$$
\begin{aligned}
F\left(x_{\varepsilon}, t_{\varepsilon}, p_{\varepsilon}, \Lambda_{p_{\varepsilon}, f_{u}}\left[\psi_{u}\right]\left(x_{\varepsilon}^{\prime}\right)\right)-F\left(y_{\varepsilon}, s_{\varepsilon}, p_{\varepsilon}, \bar{\Lambda}_{p_{\varepsilon}, f_{v}}\left[\psi_{v}\right]\left(y_{\varepsilon}^{\prime}\right)\right) \\
\geq \\
\quad F\left(x_{\varepsilon}, t_{\varepsilon}, p_{\varepsilon}, \bar{\Lambda}_{p_{\varepsilon}, f_{v}}\left[\psi_{v}\right]\left(y_{\varepsilon}^{\prime}\right)+L_{f}\left|x_{\varepsilon}-y_{\varepsilon}\right|+\omega_{f}\left(\left|t_{\varepsilon}-s_{\varepsilon}\right|\right)\right) \\
\quad-F\left(y_{\varepsilon}, s_{\varepsilon}, p_{\varepsilon}, \bar{\Lambda}_{p_{\varepsilon}, f_{v}}\left[\psi_{v}\right]\left(y_{\varepsilon}^{\prime}\right)\right) \\
=: I .
\end{aligned}
$$

If we assume (i), that is, $v$ is Lipschitz in space, uniformly in time, with Lipschitz constant $L_{v}$, we must have $\left|p_{\varepsilon}\right| \leq L_{v}$. Therefore we can find a modulus of continuity $\omega_{F}$ of $F$ on a sufficiently large bounded subset of $\mathbb{R}^{n} \times \mathbb{R} \times \mathbb{R}^{n} \times \mathbb{R}$ and we can estimate

$$
I \geq-\omega_{F}\left(\left|x_{\varepsilon}-y_{\varepsilon}\right|+\left|t_{\varepsilon}-s_{\varepsilon}\right|+L_{f}\left|x_{\varepsilon}-y_{\varepsilon}\right|+\omega_{f}\left(\left|t_{\varepsilon}-s_{\varepsilon}\right|\right)\right),
$$

where the right-hand side converges to 0 as $\varepsilon \rightarrow 0$.

On the other hand, if we assume (ii), that is, that $F$ satisfies (3.1) and $f$ is Lipschitz in both variables, we can estimate

$$
\begin{aligned}
I & \geq-C\left(L_{f}+1\right)\left(\left|p_{\varepsilon}\right|+1\right)\left(\left|x_{\varepsilon}-y_{\varepsilon}\right|+\left|t_{\varepsilon}-s_{\varepsilon}\right|\right) \\
& \geq-K\left(\left|p_{\varepsilon}\right|+1\right) \sqrt{\varepsilon}
\end{aligned}
$$

for some $K$ independent of $\varepsilon$, where we used (3.3). But $\left|p_{\varepsilon}\right| \sqrt{\varepsilon} \rightarrow 0$ as $\varepsilon \rightarrow 0$ by (3.4) and so the right-hand side converges to 0 as $\varepsilon \rightarrow 0$.

Either way, since by (3.7) we have

$$
I \leq-\frac{\mu}{T^{2}}<0,
$$

we arrive at a contradiction. We conclude that $\sup u-v \leq 0$. This finishes the proof of the comparison principle.

\section{Stability}

In this section we assume that $F$ does not depend on the $x$ variable explicitly, and consider the stability of viscosity solutions of (2.6) with respect to an approximation by solutions of regularized problems

$$
u_{t}+F\left(t, \nabla u, \operatorname{div}\left(\nabla \sigma_{m}(\nabla u)\right)-f\right)=0
$$

as an extension of the proof in [21]. Recall that we consider two modes of regularization of $\sigma$ :

(a) $\quad \sigma_{m} \in C^{2}\left(\mathbb{R}^{n}\right), a_{m}^{-1} \leq \nabla^{2} \sigma_{m} \leq a_{m}$ for some $a_{m}>0$, and $\sigma_{m} \searrow \sigma$; or

(b) $\sigma_{m}$ is an anisotropy, $\sigma_{m} \in C^{2}\left(\mathbb{R}^{n} \backslash\{0\}\right),\left\{\sigma_{m}<1\right\}$ is strictly convex, and $\sigma_{m} \rightarrow \sigma$ locally uniformly.

We have the following theorem for the approximation scheme (a). 
Theorem 4.1 Let $\sigma$ be a crystalline anisotropy, $F \in C\left(\mathbb{R} \times \mathbb{R}^{n} \times \mathbb{R}\right)$ be non-increasing in the last variable, and $f \in C\left(\mathbb{R}^{n} \times \mathbb{R}\right)$ be Lipschitz continuous in space, uniformly in time. Let $\left\{u_{m}\right\}$ be a locally bounded sequence of viscosity subsolutions of (4.1) on an open set $U \subset \mathbb{R}^{n} \times \mathbb{R}$ with $\sigma_{m}$ as in (a). Then lim sup ${ }_{m \rightarrow \infty} u_{m}$ is a viscosity subsolution of (2.6) on $U$. Similarly, if $u_{m}$ are viscosity supersolutions then $\liminf _{* m \rightarrow \infty} u_{m}$ is a viscosity supersolution of (2.6).

Remark 4.2 We can also allow for $f$ to be locally uniformly approximated by a sequence $f_{m}$ with a uniform Lipschitz constant in space, and for $F$ to be locally uniformly approximated by continuous functions $F_{m}$, nonincreasing in the last variable. This will only figure in (4.2) where we need to add a subscript $m_{l}$ to $F$ and $f$. Due to the locally uniform convergence we recover (4.3).

Remark 4.3 Note that unlike in the comparison principle, Theorem 3.1, we cannot allow $F$ to explicitly depend on the space variable $x$. Indeed, in the proof below, we only know that the accumulation points of $\left\{x_{a}\right\}_{a>0}$ lie in the set $N \ni \hat{x}$, and it is not clear that $\hat{x}$ is one of them to deduce (4.5). Note that a typical perturbation like adding $|x|^{4}$ to $\varphi$ does not work since this does not yield an admissible faceted test function.

For the approximating scheme (b) by anisotropies $\sigma_{m}$ we need to assume that the approximating sequence $\left\{u_{m}\right\}$ are solutions with uniformly bounded continuous initial data.

Theorem 4.4 Let $\sigma, F$ and $f$ be as in Theorem 4.1. Let $T>0$ and let $u_{m}$ be a locally bounded sequence of viscosity solutions of (4.1) on $\mathbb{R}^{n} \times(0, T)$ with $\sigma_{m}$ as in (b) with initial data $u_{m}(\cdot, 0)=u_{0, m}$, where $u_{0, m} \in C\left(\mathbb{R}^{n}\right)$ are uniformly bounded. Then limsup ${ }_{m \rightarrow \infty}{ }_{m \rightarrow}$ is a viscosity subsolution of (2.6) and $\liminf _{* m \rightarrow \infty} u_{m}$ is a viscosity supersolution of (2.6) on $\mathbb{R}^{n} \times(0, T)$.

Proof As in [21], we approximate each $u_{m}$ by solutions $u_{m, \delta}$ of (4.1) with initial data $u_{0, m}$ and $\sigma_{m, \delta}$ smooth with quadratic growth, as in (a) in the limit $\delta \searrow 0$. The rest follows the proof of Theorem 4.1. We refer the reader to [21] for further details.

\subsection{Proof of Theorem 4.1}

Let

$$
u=\limsup _{m \rightarrow \infty} u_{m}
$$

We follow the approach in [22]. We start with the simpler setting when $\hat{p}=0$ and $f=f(x)$ to explain the structure of the proof.

Suppose that a 0 -admissible test function is of the form $\varphi(x, t)=\psi(x)+g(t)$ where $\psi$ is 0 -admissible with facet containing 0 in its interior, and suppose that $u-\varphi(\cdot-h, \cdot)$ has a global maximum 0 at $(x, t)=(0,0)$ for all $|h| \leq \rho$ for some fixed $\rho>0$. By Remark 2.2, we can modify $\psi$ away from the facet $\{\psi=0\}$ by distance greater than $2 \rho$ so that $\psi$ is a periodic Lipschitz function with a Cahn-Hoffman vector field. We also modify $f$ away from the facet so that it is periodic and Lipschitz. By rescaling, we can assume that $\psi$ is $\mathbb{Z}^{n}$ 
periodic so that $\psi \in \operatorname{Lip}\left(\mathbb{T}^{n}\right)$. We have $\partial \mathcal{E}_{f}(\psi) \neq \emptyset$ where $\mathcal{E}_{f}$ is defined as in (1.4) over $\mathbb{T}^{n}$. Since $\Lambda_{0, f}[\psi]=-\partial^{0} \mathcal{E}_{f}(\psi)$ on $\{\psi=0\}$, we can approximate it by the resolvent problems for $\mathcal{E}_{f}$ and $\mathcal{E}_{m, f}$,

$$
\psi_{a}+a \partial \mathcal{E}_{f}\left(\psi_{a}\right) \ni \psi, \quad \psi_{a, m}+a \partial \mathcal{E}_{m, f}\left(\psi_{a, m}\right) \ni \psi .
$$

Since the resolvent problem for $\mathcal{E}_{m, f}$ is uniformly elliptic, standard regularity yields $\psi_{a, m} \in C^{2}\left(\mathbb{T}^{n}\right)$. Moreover, $\psi_{a}$ and $\psi_{a, m}$ are Lipschitz continuous with Lipschitz constant $L+a L_{f}$, where $L$ is the Lipschitz constant of $\psi$ and $L_{f}$ is the Lipschitz constant of $f$; see Section A. Due to the Mosco convergence of $\mathcal{E}_{m, f}$ to $\mathcal{E}_{f}$, we deduce the convergence $\psi_{a, m} \rightarrow \psi_{a}$ in $L^{2}$. Therefore the convergence is uniform due to the Lipschitz continuity. Finally, $\left(\psi_{a}-\psi\right) / a \rightarrow-\partial^{0} \mathcal{E}_{f}(\psi)$ in $L^{2}\left(\mathbb{T}^{n}\right)$.

We define the $\rho$-neighborhood of the facet,

$$
O:=\{x: \psi(y)=0 \text { for some } y,|y-x| \leq \rho\} .
$$

Note that we did not modify $\psi$ above closer than $2 \rho$ from the facet $\{\psi=0\}$ and so $\psi(\cdot-w)$ was not modified on $O$ for all $|w| \leq \rho$. We define

$$
\bar{u}(x):=\sup _{|t| \leq \rho}[u(x, t)-g(t)] .
$$

We have $\bar{u}-\psi(\cdot-w) \leq 0$ on $O$ for $|w| \leq \rho$, with equality at $x=0$.

For $\delta:=\rho / 5$, we define the set

$$
N:=\{x \in O: \bar{u}(x) \geq 0, \psi(x-w) \leq 0 \text { for some }|w| \leq \delta\} .
$$

Note that $N$ is bounded since $O$ is bounded. Since $\bar{u}-\psi(x-z)$ has a maximum 0 for any $|z| \leq \rho=5 \delta$, we showed in [21, Corollary 8.3] that

$$
\bar{u}(x) \leq 0 \quad \text { and } \quad \psi(x-z) \geq 0 \quad \text { for all } \operatorname{dist}(x, N) \leq 3 \delta,|z| \leq \delta,
$$

and $\operatorname{dist}(N, \partial O) \geq 4 \delta$.

The key consequence is that we can modify $\psi$ away from the facet while preserving its sign and the fact that all points of maxima of $\bar{u}-\psi(\cdot-z)$ in $3 \delta$-neighborhood of $N$ all lie in $N$, whenever $|z| \leq \delta$. In particular, we can assume that $\psi$ has arbitrarily small Lipschitz constant $L>0$ by simply multiplying $\psi$ by a small positive number. It is convenient to introduce $M^{s}$, the $s$-neighborhood of $N \times\{0\}$, as

$$
M^{s}:=\{(x, t): \operatorname{dist}(x, N) \leq s,|t| \leq s\} .
$$

By adding $|t|^{2}$ to $g(t)$, we may also assume that $u-\varphi(\cdot-z, \cdot)$ takes on its maximum 0 in the set $M^{3 \delta}$ at $t=0$, whenever $|z| \leq \delta$.

To be able to conclude (4.4) below, for every $a>0$ we choose $z_{a}$ with $\left|z_{a}\right| \leq \delta$ satisfying $\psi_{a}\left(z_{a}\right)=\min _{|w| \leq \delta} \psi_{a}(w)$. By the uniform convergence of $\psi_{a} \rightarrow \psi$, for all $a$ small enough all the maxima of $u-\varphi_{a}\left(\cdot+z_{a}, \cdot\right)$ in $M^{3 \delta}$ lie in $M^{\delta}$, that is, $\operatorname{argmax}_{M^{3 \delta}}\left[u-\varphi_{a}\left(\cdot+z_{a}, \cdot\right)\right] \subset M^{\delta}$. For any such small $a$ there exists a point of maximum $\left(x_{a}, t_{a}\right)$ in $M^{\delta}$ and a sequence $m_{\ell} \rightarrow \infty$ and a sequence $\left(x_{\ell}, t_{\ell}\right) \rightarrow\left(x_{a}, t_{a}\right)$ of points of maxima of $u_{m_{\ell}}-\varphi_{a, m_{\ell}}\left(\cdot+z_{a}, \cdot\right)$ in $M^{3 \delta}$. Since $\psi_{a, m}$ are Lipschitz continuous with Lipschitz constant $L+a L_{f}$, we can also assume that $\nabla \psi_{a, m_{\ell}} \rightarrow p_{a}$ as $\ell \rightarrow \infty$ for some $\left|p_{a}\right| \leq L+a L_{f}$.

The definition of the viscosity subsolution $u_{m}$ implies 


$$
g^{\prime}\left(t_{\ell}\right)+F\left(t_{\ell}, \nabla \psi_{a, m_{\ell}}\left(x_{\ell}+z_{a}\right), \operatorname{div}\left(\nabla \sigma_{m_{\ell}}\left(\nabla \psi_{a, m_{\ell}}\right)\right)\left(x_{\ell}+z_{a}\right)-f\left(x_{\ell}\right)\right) \leq 0
$$

But $\operatorname{div}\left(\nabla \sigma_{m_{\ell}}\left(\nabla \psi_{a, m_{\ell}}\right)\right)-f=-\partial^{0} \mathcal{E}_{m_{\ell} f}\left(\psi_{a, m_{\ell}}\right)=\left(\psi_{a, m_{\ell}}-\psi\right) / a$, and so by the uniform convergence $\psi_{a, m_{\ell}} \rightarrow \psi_{a}$ we recover in the limit $\ell \rightarrow \infty$ the inequality

$$
g^{\prime}\left(t_{a}\right)+F\left(t_{a}, p_{a}, \frac{\psi_{a}\left(x_{a}+z_{a}\right)-\psi\left(x_{a}+z_{a}\right)}{a}+f\left(x_{a}+z_{a}\right)-f\left(x_{a}\right)\right) \leq 0 .
$$

Due to the choice of $z_{a}$ and [21, Lemma 8.5], we have

$$
\frac{\psi_{a}-\psi}{a}\left(x_{a}+z_{a}\right) \leq \min _{|w| \leq \delta} \frac{\psi_{a}-\psi}{a}(w) .
$$

The monotonicity of $F$ in the last variable, uniform continuity of $F$ on compact sets and the $L_{f}$-Lipschitz continuity of $f$ yields

$$
g^{\prime}\left(t_{a}\right)+F\left(t_{a}, p_{a}, \min _{|w| \leq \delta} \frac{\psi_{a}-\psi}{a}(w)\right) \leq \omega_{F}\left(L_{f}\left|z_{a}\right|\right),
$$

for a modulus of continuity $\omega_{F}$. We now find a sequence $a_{j} \rightarrow 0$ such that $p_{a_{j}} \rightarrow p$ for some $|p| \leq L$, and

$$
\lim _{j \rightarrow \infty} \min _{|w| \leq \delta} \frac{\psi_{a_{j}}-\psi}{a_{j}}(w)=\liminf _{a \searrow 0} \min _{|w| \leq \delta} \frac{\psi_{a}-\psi}{a}(w) .
$$

Since $t_{a_{j}} \rightarrow 0=\hat{t}$, we recover

$$
g^{\prime}(0)+F\left(0, p, \liminf _{a \searrow 0} \min _{|w| \leq \delta} \frac{\psi_{a}-\psi}{a}(w)\right) \leq \omega_{F}\left(L_{f} \delta\right) .
$$

And since $\left(\psi_{a}-\psi\right) / a \rightarrow \Lambda_{0, f}[\psi]$ in $L^{2}\left(B_{\delta}(0)\right)$, we have by the monotonicity of $F$ in the last variable

$$
g^{\prime}(0)+F\left(0, p, \operatorname{ess} \inf _{|w| \leq \delta} \Lambda_{0, f}[\psi](w)\right) \leq \omega_{F}\left(L_{f} \delta\right) .
$$

As we explained above, $L>0$ can be taken arbitrarily small, $|p| \leq L$ and so by continuity

$$
g^{\prime}(0)+F\left(0,0, \operatorname{ess} \inf _{|w| \leq \delta} \Lambda_{0, f}[\psi](w)\right) \leq \omega_{F}\left(L_{f} \delta\right) .
$$

Since we can take $\rho>0$ and therefore $\delta>0$ arbitrarily small, we deduce (2.7). We have verified (2.7) for $\hat{p}=0$.

In the case of general $\hat{p}$, and $f=f(x, t)$, we have a more complicated $\hat{p}$-admissible test function $\varphi(x, t)=\psi\left(x^{\prime}\right)+\theta\left(x^{\prime \prime}\right)+\hat{p} \cdot x+g(t)$. Let $k, Z, \mathcal{T}$ and $\mathcal{T}_{\perp}$ correspond to $\hat{p}$. Suppose that $u-\varphi(\cdot-\mathcal{T} w, \cdot)$ has a global max at $(\hat{x}, \hat{t})$ for small $w \in \mathbb{R}^{k}$. By translation, we may assume that $(\hat{x}, \hat{t})=(0,0)$. By rotating the coordinate system, we may assume that $Z=\mathbb{R}^{k} \times\{0\}, Z^{\perp}=\{0\} \times \mathbb{R}^{n-k}$ so that $x=\left(x^{\prime}, x^{\prime \prime}\right), x^{\prime} \in \mathbb{R}^{k}, x^{\prime \prime} \in \mathbb{R}^{n-k}$.

We proceed as in [21]. First, recalling [21, Corollary 8.3], we note that by adding $\left|x^{\prime \prime}\right|^{2}$ to $\theta\left(x^{\prime \prime}\right)$ and $|t|^{2}$ to $g(t)$, we may assume that the maximum of $u-\varphi(\cdot-\mathcal{T} w, \cdot)$ in $M^{3 \delta}$ is attained only on $M^{0}=N \times\{0\} \times\{0\}$ for any small $w$, where 


$$
M^{s}:=\left\{(x, t): \operatorname{dist}\left(x^{\prime}, N\right) \leq s,\left|x^{\prime \prime}\right| \leq s,|t| \leq s\right\} .
$$

By the above mentioned corollary and $\nabla \theta(0)=0$, we may also assume that the Lipschitz constant of $\psi$ and $\theta$ on $M^{3 \delta}$ is smaller than any chosen fixed $L>0$. By modifying $\psi$ away from $N$ and $\theta$ away from 0 , we can make them periodic, while preserving the Lipschitz constant $L$. As $N$ is bounded, scaling allows us to assume that they are 1 periodic and that $\operatorname{diam}(N)<1$. This way we can again consider a resolvent problem on $\mathbb{T}^{n}$, but with the reduced energy

$$
\overline{\mathcal{E}}(v):=\int \bar{\sigma}(D v)+\bar{f} v, \quad v \in B V\left(\mathbb{T}^{n}\right) \cap L^{2}\left(\mathbb{T}^{n}\right),
$$

where

$$
\bar{\sigma}(p)=\lim _{\lambda \searrow 0} \frac{\sigma(\hat{p}+\lambda p)-\sigma(\hat{p})}{\lambda},
$$

and

$$
\bar{f}(x):=f\left(\mathcal{T} x^{\prime}, 0\right), \quad \operatorname{dist}\left(x^{\prime},\{\psi=0\}\right) \text { small, }
$$

and $\bar{f}$ is extended periodically away from the facet in the directions in $Z$. Note that $\bar{\sigma}$ is linear on $Z^{\perp}$, and $\bar{f}$ is constant in the directions in $Z^{\perp}$. In fact, $\mathcal{T} x^{\prime}=\mathcal{T} \mathcal{T}^{*} x$ is the orthogonal projection on $Z$. $D v$ denotes the vector-valued Radon measure that is the derivative of a $B V$ function $v$, in contrast to $\nabla v$, which often denotes only the absolutely continuous part with respect to the Lebesgue measure. One way to understand the definition of $\overline{\mathcal{E}}(v)$ is to define it for $W^{1,1} \cap L^{2}$ functions with $\nabla v$ and take the lower semicontinuous envelope with respect to the $L^{2}$ convergence.

As we showed in [21, Lemma 3.9], the solution $\bar{\psi}_{a}$ of the resolvent problem

$$
\bar{\psi}_{a}+a \partial \overline{\mathcal{E}}\left(\bar{\psi}_{a}\right) \ni \bar{\psi}
$$

with $\bar{\psi}(x)=\psi\left(x^{\prime}\right)+\theta\left(x^{\prime \prime}\right)$ is of the form

$$
\bar{\psi}_{a}(x)=\psi_{a}\left(x^{\prime}\right)+\theta\left(x^{\prime \prime}\right),
$$

where $\psi_{a}$ is the solution of the sliced resolvent problem

$$
\psi_{a}+a \partial \mathcal{E}^{\mathrm{sl}}\left(\psi_{a}\right) \ni \psi
$$

with the sliced energy

$$
\mathcal{E}^{\mathrm{sl}}(v):=\int \sigma_{\hat{p}}^{\mathrm{sl}}(D v)+\hat{f} v, \quad v \in B V\left(\mathbb{T}^{k}\right) \cap L^{2}\left(\mathbb{T}^{k}\right),
$$

with $\hat{f}(w):=\bar{f}(\mathcal{T} w)$. We recall that

$$
\frac{\psi_{a}-\psi}{a} \rightarrow \Lambda_{\hat{p}, \hat{f}} \quad \text { as } a \rightarrow 0 \text { in } L^{2}(\{\psi=0\}) .
$$

Let $\bar{\psi}_{a, m}$ be the solution of the resolvent problem 


$$
\bar{\psi}_{a, m}+a \partial \overline{\mathcal{E}}_{m}\left(\bar{\psi}_{a, m}\right) \ni \bar{\psi}
$$

where

$$
\overline{\mathcal{E}}_{m}(v):=\int \sigma_{m}(\nabla v+\hat{p})+\bar{f} v, \quad v \in H^{1}\left(\mathbb{T}^{n}\right) .
$$

We recall that $\overline{\mathcal{E}}_{m}$ Mosco-converges to the energy $\int \sigma(D v+\hat{p})+\bar{f} v$, which is equal to $\overline{\mathcal{E}}$ for functions with sufficiently small Lipschitz constant since $\sigma$ and $\bar{\sigma}$ differ by a constant on a small neighborhood of $\hat{p}$ depending only on $\hat{p}$ since $\sigma$ is crystalline. Thus we have $\bar{\psi}_{a, m} \rightrightarrows \bar{\psi}_{a}$ as long as $L, a>0$ are taken sufficiently small. For more details, see [21].

For each $a>0$, we select $z_{a} \in \mathbb{R}^{k}$ with

$$
\psi_{a}\left(z_{a}\right)=\min _{|w| \leq \delta} \psi_{a}(w) .
$$

For a fixed $a>0$, there exist a sequence of points of maximum $\left(x_{a, m}, t_{a, m}\right)$ of $u_{m}(x, t)-$ $\bar{\psi}_{a, m}\left(x+\mathcal{T} z_{a}\right)-\hat{p} \cdot x-g(t)$ on $M^{3 \delta}$ and a point of maximum $\left(x_{a}, t_{a}\right)$ of $u(x, t)-\psi_{a}\left(x^{\prime}+\right.$ $\left.z_{a}\right)-\theta\left(x^{\prime \prime}\right)-\hat{p} \cdot x-g(t)$ on $M^{3 \delta}$ such that $\left(x_{a, m}, t_{a, m}\right) \rightarrow\left(x_{a}, t_{a}\right)$ as $m \rightarrow \infty$ along a subsequence.

By the viscosity subsolution condition for (4.1) we have

$$
\begin{aligned}
& g^{\prime}\left(t_{a, m}\right)+F\left(t_{a, m}, \nabla \bar{\psi}_{a, m}\left(x_{a}\right)+\hat{p},\right. \\
& \left.\quad \operatorname{div} \nabla \sigma_{m}\left(\nabla \bar{\psi}_{a, m}+\hat{p}\right)\left(x_{a, m}+\mathcal{T} z_{a}\right)-f\left(x_{a, m}, t_{a, m}\right)\right) \leq 0 .
\end{aligned}
$$

By the Lipschitz bound for $\psi_{a, m}$, there is $p_{a}$ such that $\nabla \bar{\psi}_{a, m}\left(x_{a, m}\right) \rightarrow p_{a}$ along a subsequence $\quad m \rightarrow \infty$. We also have $\left|p_{a}\right| \leq L+a L_{f}$. Since $\operatorname{div} \nabla \sigma_{m}\left(\nabla \bar{\psi}_{a, m}+\hat{p}\right)=\left(\bar{\psi}_{a, m}-\bar{\psi}\right) / a+\bar{f}$, continuity and uniform convergence yield along the subsequence $m \rightarrow \infty$ that

$$
\begin{aligned}
& g^{\prime}\left(t_{a}\right)+F\left(t_{a}, p_{a}+\hat{p},\right. \\
& \left.\quad\left(\psi_{a}\left(x_{a}^{\prime}+z_{a}\right)-\psi\left(x_{a}^{\prime}+z_{a}\right)\right) / a+\bar{f}\left(x_{a}+\mathcal{T} z_{a}\right)-f\left(x_{a}, t_{a}\right)\right) \leq 0 .
\end{aligned}
$$

Now we estimate

$$
\begin{aligned}
\bar{f}\left(x_{a}+\mathcal{T} z_{a}\right)-f\left(x_{a}, t_{a}\right) & =f\left(\mathcal{T}\left(x_{a}^{\prime}+z_{a}\right), 0\right)-f\left(\mathcal{T} x_{a}^{\prime}+\mathcal{T}_{\perp} x_{a}^{\prime \prime}, t_{a}\right) \\
& \leq \omega_{f}\left(\left|x_{a}^{\prime \prime}\right|+\left|t_{a}\right|+\left|z_{a}\right|\right),
\end{aligned}
$$

where $\omega_{f}$ is a modulus of continuity of $f$ on a sufficiently large subset of $\mathbb{R}^{n} \times \mathbb{R}$. We use the above estimate and [21, Lemma 8.5] to deduce

$$
g^{\prime}\left(t_{a}\right)+F\left(t_{a}, p_{a}+\hat{p}, \min _{|w| \leq \delta}\left(\psi_{a}(w)-\psi(w)\right) / a\right) \leq \omega_{F}\left(\omega_{f}\left(\left|x_{a}^{\prime \prime}\right|+\left|t_{a}\right|+\delta\right)\right),
$$

where $\omega_{F}$ is a modulus of continuity of $F$ on a sufficiently large subset of $\mathbb{R} \times \mathbb{R}^{n} \times \mathbb{R}$. Now sending $a \searrow 0$ along a subsequence so that $p_{a} \rightarrow p$ for some $p$ with $|p| \leq L$ and using that $\left(\psi_{a}-\psi\right) / a \rightarrow \Lambda_{\hat{p}, f}[\psi]$ in $L^{2}\left(B_{\delta}(0)\right),\left|x_{a}^{\prime \prime}\right| \rightarrow 0$ and $\left|t_{a}\right| \rightarrow 0$, we recover

$$
g^{\prime}(0)+F\left(0, p+\hat{p}, \underset{|w| \leq \delta}{\operatorname{ess} \inf } \Lambda_{\hat{p}, f}[\psi](w)\right) \leq \omega_{F}\left(\omega_{f}(\delta)\right)
$$


Since we can take $L>0$ and $\delta>0$ as small as we want, we deduce

$$
g^{\prime}(0)+F\left(0, \hat{p}, \underline{\Lambda}_{\hat{p}, \hat{f}}[\psi](0)\right) \leq 0
$$

Therefore the viscosity solution condition (2.7) is satisfied for any $\hat{p}$-admissible test function.

This shows that $u$ is a viscosity subsolution of (2.6). Since the proof that $\lim _{\inf _{* m \rightarrow \infty}} u_{m}$ is a viscosity supersolution of (2.6) is analogous, this finishes the proof of Theorem 4.1.

\section{Lipschitz bound}

In this section we show that the solutions of the approximating problems (4.1) have a modulus of continuity uniform in $m$. We consider a level set equation of $V=g\left(v, \kappa_{\sigma}+f\right)$ of the form

$$
u_{t}+|\nabla u| g(\nabla u /|\nabla u|,-\operatorname{div}(\nabla \sigma(\nabla u))+f(x, t))=0 \quad \text { in } \quad \mathbb{R}^{n} \times(0, T), \quad T>0,
$$

where the anisotropy $\sigma$ is smooth. For the following Lipschitz estimate we do not need to assume that $\sigma$ is positively one-homogeneous.

Theorem 5.1 Assume that $\sigma \in C^{2}\left(\mathbb{R}^{n} \backslash\{0\}\right)$ is convex, $g \in C\left(\mathcal{S}^{n-1} \times \mathbb{R}\right)$ is Lipschitz in the second variable with a Lipschitz constant $L_{g}$ uniform in the first variable, $g$ is nondecreasing in the second variable, and $f \in C\left(\mathbb{R}^{n} \times[0, T]\right)$ is Lipschitz in space with a Lipschitz constant $L_{f}$ uniform in time. Let $u \in C\left(\mathbb{R}^{n} \times[0, T]\right)$ be a solution of (5.1) in $\mathbb{R}^{n} \times(0, T)$. Assume that $u$ is constant outside $B \times[0, T]$, where $B$ is a (large) ball. If the initial data $u_{0}$ is Lipschitz, then

$$
|u(x, t)-u(y, t)| \leq L e^{M t}|x-y|, \quad x, y \in \mathbb{R}^{n}, \quad t \in[0, T]
$$

with $M=L_{g} L_{f}, L=\operatorname{Lip}\left(u_{0}\right)$.

Proof Suppose that the conclusion were false. Then,

$$
m_{0}:=\sup _{\substack{Q \times Q \\ t=s}} \Phi(x, t, y, s)>0, \quad \Phi(x, t, y, s):=u(x, t)-u(y, s)-L e^{M t}|x-y|,
$$

where $Q=\mathbb{R}^{n} \times[0, T]$. By uniform continuity of $u$ in $Q$, for any $\varepsilon>0$ there is $\delta>0$ such that $|t-s| \leq \delta$ implies that $|u(x, t)-u(x, s)|<\varepsilon$ for all $x \in \mathbb{R}^{n}$. We fix $\varepsilon=m_{0} / 8$ so that

$$
\sup _{\substack{Q \times Q \\|t-s| \leq \delta}} \Phi(x, t, y, s) \geq m_{0} / 2 .
$$

We take $\beta>0$ sufficiently small and fix it so that

$$
m_{1}:=\sup _{\substack{Q \times Q \\|t-s| \leq \delta}}(\Phi(x, t, y, s)-\beta /(T-t)) \geq m_{0} / 4 .
$$

For $\alpha>1$, we consider

$$
\Psi(x, t, y, s):=\Phi(x, t, y, s)-\beta /(T-t)-\alpha(t-s)^{2} .
$$


Since $u$ is continuous and is a constant on $\left(\mathbb{R}^{n} \backslash B\right) \times[0, T]$ with a big ball $B$, there is a maximizer $\left(x_{\alpha}, t_{\alpha}, y_{\alpha}, s_{\alpha}\right) \in Q \times Q$ of $\Psi$. Moreover, if $\alpha$ is sufficiently large, say $\alpha>\alpha_{0}$, then $\left|t_{\alpha}-s_{\alpha}\right|<\delta$. This yields

$$
m_{1} \geq \max _{Q \times Q} \Psi(x, t, y, s), \quad c_{0}:=\sup _{\alpha>\alpha_{0}} 1 /\left|x_{\alpha}-y_{\alpha}\right|<\infty
$$

by uniform continuity of $u$ in $Q$. Moreover, we see that $t_{\alpha}-s_{\alpha} \rightarrow 0$ as $\alpha \rightarrow \infty$ since $m_{1}>0$. Since $L=\operatorname{Lip}\left(u_{0}\right)$, we observe that $\Phi(x, 0, y, 0) \leq 0$ for all $x, y \in \mathbb{R}^{n}$. Thus any accumulation point of $\left\{t_{\alpha}\right\}$ should not be zero since $t_{\alpha}-s_{\alpha} \rightarrow 0$. We may assume that $t_{\alpha}, s_{\alpha}$ are away from zero for sufficiently large $\alpha$, say $\alpha>\alpha_{1} \geq \alpha_{0}$. Evidently, $t_{\alpha}, s_{\alpha}<T$. We rewrite $\Psi$ as

$$
\begin{aligned}
& \Psi(x, t, y, s)=u(x, t)-u(y, s)-\varphi(x, t, y, s), \\
& \varphi(x, t, y, s):=\alpha(t-s)^{2}+\beta /(T-t)+L e^{M t}|x-y| .
\end{aligned}
$$

Our maximizer $z_{\alpha}=\left(x_{\alpha}, t_{\alpha}, y_{\alpha}, s_{\alpha}\right)$ should be in the interior of $Q \times Q$.

We are now in position to apply a parabolic version of Crandall-Ishii's lemma [7, 12, Theorem 3.3.3]. It provides $n \times n$ symmetric matrices $X_{\alpha}, Y_{\alpha}$ such that

$$
\left(\varphi_{t}\left(z_{\alpha}\right), \nabla_{x} \varphi\left(z_{\alpha}\right), X_{\alpha}\right), \quad\left(\operatorname{resp} .\left(-\varphi_{s}\left(z_{\alpha}\right),-\nabla_{y} \varphi\left(x_{\alpha}\right),-Y_{\alpha}\right)\right)
$$

can be approximated by super (resp. sub) parabolic semijets of $u$ at $\left(x_{\alpha} t_{\alpha}\right)$ (resp. $\left(y_{\alpha}, s_{\alpha}\right)$ ). Moreover, $X_{\alpha}+Y_{\alpha} \leq 0$ and the norms $\left\|X_{\alpha}\right\|,\left\|Y_{\alpha}\right\|$ are bounded by the spatial second derivatives of $\varphi$ at $z_{\alpha}$ for $\alpha>\alpha_{1}$ which are bounded since $c_{0}<\infty$. Since $u$ is a sub- and supersolution and $\nabla_{x} \varphi\left(z_{\alpha}\right)=-\nabla_{y} \varphi\left(z_{\alpha}\right)=: p_{\alpha}$, we end up with

$$
\begin{aligned}
& \varphi_{t}+\left|p_{\alpha}\right| g\left(p_{\alpha} /\left|p_{\alpha}\right|,-\operatorname{tr}\left(\nabla^{2} \sigma\left(p_{\alpha}\right) X_{\alpha}\right)+f\left(x_{\alpha}, t_{\alpha}\right)\right) \leq 0 \quad \text { at } \quad z_{\alpha} \\
& \quad-\varphi_{s}+\left|p_{\alpha}\right| g\left(p_{\alpha} /\left|p_{\alpha}\right|,-\operatorname{tr}\left(\nabla^{2} \sigma\left(p_{\alpha}\right)\left(-Y_{\alpha}\right)\right)+f\left(y_{\alpha}, s_{\alpha}\right)\right) \geq 0 \quad \text { at } \quad z_{\alpha} .
\end{aligned}
$$

In the second inequality, we may replace $-Y_{\alpha}$ by $X_{\alpha}$ since $X_{\alpha}+Y_{\alpha} \leq 0$ and $g$ is monotone in the last variable (ellipticity). The resulting inequality is

$$
-\varphi_{s}+\left|p_{\alpha}\right| g\left(p_{\alpha} /\left|p_{\alpha}\right|,-\operatorname{tr}\left(\nabla^{2} \sigma\left(p_{\alpha}\right) X_{\alpha}\right)+f\left(y_{\alpha}, s_{\alpha}\right)\right) \geq 0 \quad \text { at } \quad z_{\alpha} .
$$

Since

$$
\left|g\left(p, \xi_{1}\right)-g\left(p, \xi_{2}\right)\right| \leq L_{g}\left|\xi_{1}-\xi_{2}\right|,
$$

subtracting (5.4) from (5.3) yields

$$
\varphi_{t}\left(z_{\alpha}\right)+\varphi_{s}\left(z_{\alpha}\right)-\left|p_{\alpha}\right| L_{g}\left|f\left(x_{\alpha} t_{\alpha}\right)-f\left(y_{\alpha}, s_{\alpha}\right)\right| \leq 0
$$

By a simple manipulation, we get

$$
\begin{aligned}
\varphi_{t}\left(z_{\alpha}\right) & =\beta /\left(T-t_{\alpha}\right)^{2}+L M e^{M t_{\alpha}}\left|x_{\alpha}-y_{\alpha}\right|+2 \alpha\left(t_{\alpha}-s_{\alpha}\right), \\
\varphi_{s}\left(z_{\alpha}\right) & =-2 \alpha\left(t_{\alpha}-s_{\alpha}\right), \\
p_{\alpha} & =L e^{M t_{\alpha}}\left(x_{\alpha}-y_{\alpha}\right) /\left|x_{\alpha}-y_{\alpha}\right| \text { so that }\left|p_{\alpha}\right|=L e^{M t_{\alpha}} .
\end{aligned}
$$

Since $f$ is uniformly continuous in $B \times[0, T]$ and spatially Lipschitz uniformly in time, 


$$
\left|f\left(x_{\alpha}, t_{\alpha}\right)-f\left(y_{\alpha}, s_{\alpha}\right)\right| \leq L_{f}\left|x_{\alpha}-y_{\alpha}\right|+\omega\left(t_{\alpha}-s_{\alpha}\right),
$$

where $\omega$ is a modulus, i.e., $\omega \in C[0, \delta], \omega \geq 0$ and $\omega(0)=0$. Since $1 /\left(T-t_{\alpha}\right)^{2} \leq 1 / T^{2}$, (5.5) now yields

$$
\beta / T^{2}+L M e^{M t_{\alpha}}\left|x_{\alpha}-y_{\alpha}\right|-\left|p_{\alpha}\right| L_{g}\left(L_{f}\left|x_{\alpha}-y_{\alpha}\right|+\omega\left(t_{\alpha}-s_{\alpha}\right)\right) \leq 0 .
$$

Since $M=L_{g} L_{f}$, we see that

$$
L M e^{M t_{\alpha}}\left|x_{\alpha}-y_{\alpha}\right|-\left|p_{\alpha}\right| L_{g} L_{f}\left|x_{\alpha}-y_{\alpha}\right|=0 .
$$

Thus (5.6) implies

$$
\beta / T^{2}-\left|p_{\alpha}\right| L_{g} \omega\left(t_{\alpha}-s_{\alpha}\right) \leq 0 .
$$

Sending $\alpha \rightarrow \infty$ and using $t_{\alpha}-s_{\alpha} \rightarrow 0$, we obtain $\beta / T^{2} \leq 0$, which is a contradiction. The proof is now complete.

As an application of Lipschitz bound, we further derive a uniform Hölder continuity in time when $\sigma$ is positively one-homogeneous.

Theorem 5.2 Assume that $\sigma, g$ an $f$ are as in Theorem 5.1 and additionally assume that $\sigma$ is positively one-homogeneous. Let $u$ be the solution of $(5.1)$ in $\mathbb{R}^{n} \times(0, T)$. Assume the same hypotheses of Theorem 5.1 concerning $u$. Assume that $f$ is bounded. Then

$$
|u(x, t)-u(x, s)| \leq A|t-s|^{1 / 2}, \quad x \in \mathbb{R}^{n}, \quad t, s \in[0, T]
$$

with some constant A depending only on positive constants $L, M, T, G, \lambda_{+}, \lambda_{-}$such that

$$
\begin{gathered}
\sup _{r>0} \sup _{p \in \mathcal{S}^{n-1}} r|g(p, \pm(n-1) / r+\sup |f|)| \leq G, \\
\lambda_{-}|x| \leq \sigma(x) \leq \lambda_{+}|x| \text { for all } x \in \mathbb{R}^{n} .
\end{gathered}
$$

Proof We shall prove this theorem by constructing a barrier. We first observe that

$$
\nabla \sigma\left(\nabla \sigma^{\circ}(x)\right)=x /|x|
$$

so that $\operatorname{div} \nabla \sigma\left(\nabla \sigma^{\circ}(x)\right)=(n-1) /|x|$, where $\sigma^{\circ}(x)$ is the support function of the set $\{\sigma(x) \leq 1\}$, i.e.,

$$
\sigma^{\circ}(x)=\sup \{\langle x, y\rangle \mid \sigma(y) \leq 1\}
$$

The function $\sigma^{\circ}$ satisfies

$$
\lambda_{+}^{-1}|x| \leq \sigma^{\circ}(x) \leq \lambda_{-}^{-1}|x|
$$

and it is convex and positively one-homogeneous. (If $\nabla^{2} \sigma$ is strictly positive on $\mathcal{S}^{n-1}$, this $\sigma^{\circ}$ may not be smooth but in the sense of viscosity solutions the indentity $\operatorname{div}\left(\nabla \sigma\left(\nabla \sigma^{\circ}(x)\right)=(n-1) /|x|\right.$ still holds. $)$

For a given $s \in[0, T]$, by Theorem $5.1, u(\cdot, s)$ is Lipschitz with a Lipschitz constant $L_{s}=L e^{M s}$. For a given $\delta>0$ by Young's inequality, we have 


$$
|x| \leq \delta+|x|^{2} / 4 \delta
$$

This implies that

$$
\left(u(x, s)-u\left(x_{0}, s\right)\right) / L_{s} \leq \delta+\left|x-x_{0}\right|^{2} / 4 \delta \leq \delta+\lambda_{+}^{2} \sigma^{\circ}\left(x-x_{0}\right)^{2} / 4 \delta .
$$

If we take a constant $C$ large but depending only on $G$, we observe that

$$
v(x, t):=L_{s}\left(C(t-s) / \delta+\delta+\lambda_{+}^{2} \sigma^{\circ}\left(x-x_{0}\right)^{2} / 4 \delta\right)+u\left(x_{0}, s\right)
$$

is a supersolution with

$$
u(\cdot, s) \leq v(\cdot, s) .
$$

By the standard comparison theorem [12, Chapter 3], we conclude that

$$
u(x, t) \leq v(x, t) \quad \text { for } \quad t \geq s, \quad x \in \mathbb{R}^{n} .
$$

In particular,

$$
u\left(x_{0}, t\right) \leq L_{s}(C(t-s) / \delta+\delta)+u\left(x_{0}, s\right) .
$$

We take $\delta$ such that $\delta=(C(t-s))^{1 / 2}$ to get

$$
u\left(x_{0}, t\right)-u\left(x_{0}, s\right) \leq 2 L_{s}(C(t-s))^{1 / 2} \text { for all } t \geq s>0 .
$$

A symmetric argument yields the estimate from below. Since $x_{0}, s$ are arbitrary, this completes the proof.

We are ready to prove the existence of solutions of (1.2) for $F$ of the form (1.3).

Theorem 5.3 Let $F$ be of the form (1.3), where $g$ and $f$ are as in Theorem 5.1 for any $T>0$ and assume that $\sigma$ is a crystalline anisotropy. Then the Eq. (1.2) has a unique global viscosity solution on $\mathbb{R}^{n} \times(0, \infty)$ for any Lipschitz initial data $u_{0}$ constant outside a large ball B. Moreover, $u$ is Lipschitz continuous in space (5.2) and 1/2-Hölder continuous in time (5.7).

Proof We can approximate $\sigma$ by a sequence of positively one-homogeneous functions $\sigma_{m}$ as in Theorem 4.4, so that they all satisfy the assumptions of Theorem 5.2 with the same $\lambda_{ \pm}$. By the classical viscosity solution theory, (4.1) has a unique viscosity solution $u_{m}$ with the initial data $u_{0}$ for any $m$. Since $f$ is Lipschitz in space, by comparing $u_{m}$ with a barrier, for instance the $\sigma_{m}$-Wulff shape expanding exponentially fast with rate $L_{f}$, we see that for any $T>0$ there is a bounded set independent of $m$ such that all $u_{m}$ are equal to a constant outside of this set. Therefore we can assume that $f$ is bounded on $\mathbb{R}^{n} \times[0, T]$ for any $T>0$. We conclude that for any $T>0$ the sequence $\left\{u_{m}\right\}$ is uniformly Lipschitz in space by Theorem 5.1 and uniformly Hölder in time by Theorem 5.2. Hence any subsequence has a further subsequence that converges uniformly on $\mathbb{R}^{n} \times[0, T]$ to some continuous function $u$ with $u(\cdot, 0)=u_{0}$. This limit must be the unique viscosity solution of (1.2) by Theorem 4.4. In particular, the whole sequence converges to $u$ locally uniformly on $\mathbb{R}^{n} \times(0, \infty)$. 
Sketch of the proof of Theorem 1.1 Once we have existence, comparison and stability of solutions of (1.3), we are able to prove invariance under the change of depending variables as in $[21,22]$. This yields the uniqueness of the level set of a solution. Our existence result (Theorem 5.3) now yields the unique existence of the level set flow.

\section{Proof of nonexistence with $x$-dependent $F$}

In this section we show that we cannot allow the operator $F$ to depend on $x$ directly since in that case a solution might not exist in general. Let us consider the equation

$$
u_{t}+F\left(x,\left(\operatorname{sign} u_{x}\right)_{x}\right)=0, \quad x \in \mathbb{R}, t>0,
$$

with $F(x, \xi)=-\xi+f(x)$ and initial condition

$$
u(\cdot, 0)=0 .
$$

This corresponds to anisotropy $\sigma(p)=|p|$ for $p \in \mathbb{R}$.

Theorem 6.1 Let $f \in \operatorname{Lip}(\mathbb{R})$ with compact support, $f \geq 0$ but $f \not \equiv 0$ (see Fig. 1a). Set $L=1 / \max f$. Assume that

$$
\operatorname{supp} f \subset(-L, L) .
$$

Then there is no continuous solution with compact support.

Proof We show it by contradiction with the comparison principle, Theorem 3.1, which applies to equation (6.1).

Let $u$ be a solution of (6.1) with initial data 0 and compact support. Let us fix $\omega>L$. For given $a>0$ set

$$
v(x)=v_{a}(x):=\min (\max (a(|x|-\omega), a(L-\omega)), 0) .
$$

Note that $v$ has a facet $[-L, L]$ that contains the support of $f$. We claim that $w(x, t)=v_{a}(x)$ is a viscosity subsolution in the sense of Definition 2.7. Indeed, for any faceted test function that touches $w$ from above on the facet $[-L, L]$, we have $\Lambda \geq \frac{1}{L}$ and therefore

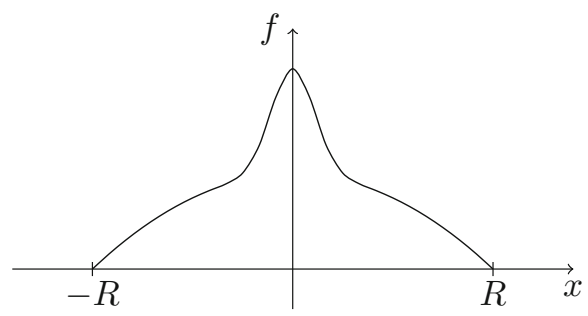

(a)

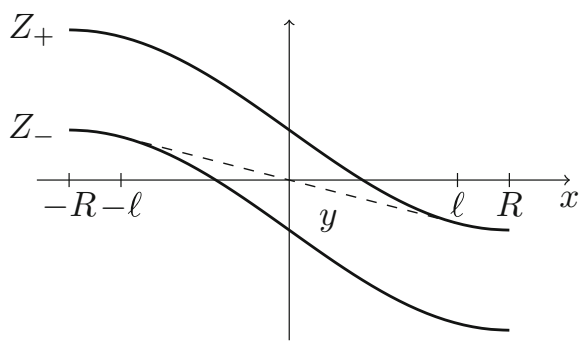

(b)

Fig. 1 a An example of the nonuniform forcing $f$ in Theorem 6.1. b The construction of $\chi_{\ell}$ 


$$
F(x, \Lambda)=-\Lambda+f \leq-\frac{1}{L}+\max f=-\max f+\max f=0 .
$$

For a test function touching anywhere else we always get $F(x, \Lambda) \leq 0$.

Therefore by the comparison principle we must have $v_{a} \leq u(\cdot, t)$ for all $t$ and $a>0$ and so $u \geq 0$. On the other hand, $f \geq 0$ and therefore $u=0$ is a supersolution so we conclude that $u \equiv 0$.

But by testing $u$ from above by a faceted test function with a facet longer than $L$, we can show that there are points where $F(x, \Lambda)=-\Lambda+f(x)>-\frac{1}{L}+f(x)=0$ and so we see that $u \equiv 0$ is not a viscosity subsolution in the sense of Definition 2.7.

In fact, this shows that a supremum of subsolutions might not be a subsolution.

One may be interested in what the solution is for $u_{t}+F\left(\left(\operatorname{sign} u_{x}\right)_{x}-f(x)\right)=0$ with $F(\xi)=-\xi$. Such a type of problems is studied in the framework of the maximal monotone operators [13]. Although a Lipschitz bound is studied only for level set equations, it is not difficult to show uniform Lipschitz bounds and also 1/2-Hölder continuity in time for the $\sigma_{m}$-approximation as we did in Sect. 5. By our convergence results we conclude that the viscosity solution agrees with that in the theory of maximum monotone operators.

We here give an explicit example of the solution. We consider $f \in \operatorname{Lip}(\mathbb{R})$ with compact support, $f \geq 0$ but $f \not \equiv 0$. To simplify the explanation we assume that $f$ is even, that is, $f(x)=f(-x), x \in \mathbb{R}$, and that $f$ is non-increasing for $f>0$. We take $R>0$ such that $\operatorname{supp} f=[-R, R]$. Let $Z_{ \pm}$be

$$
Z_{ \pm}(x)=-\int_{0}^{x} f(z) d z \pm 1 .
$$

Since $Z_{ \pm}$is convex in $x$ for $x>0$ and $Z_{ \pm}- \pm 1$ is odd, there is a unique $\ell \in(0, R)$ such that the straight segment $y=y(x)$ connecting $\left(-\ell, Z_{-}(-\ell)\right),\left(\ell, Z_{+}(\ell)\right)$ has the slope $Z_{+}^{\prime}(\ell)=-f(\ell)$; see Fig. 1b. Moreover,

$$
Z_{-}(x) \leq y(x) \leq Z_{+}(x)
$$

for $x \in[-\ell, \ell]$. By definition of $\Lambda_{f}$, we observe as in [13]

$$
\Lambda_{f}\left[\chi_{\ell}\right]=y^{\prime}(x)=\frac{Z_{+}(\ell)-Z_{-}(-\ell)}{2 \ell},
$$

if $\chi_{\ell}$ corresponds to the facet

$$
\chi_{\ell}(x):= \begin{cases}1, & x \notin[-\ell, \ell], \\ 0, & x \in[-\ell, \ell] .\end{cases}
$$

One is able to characterize $\ell$ in a slightly different way since $y^{\prime}(\ell)=-f(\ell)$. Since $\ell$ is the unique number such that

$$
-Z_{+}^{\prime}(\ell)=\frac{Z_{-}(-\ell)-Z_{+}(\ell)}{2 \ell}
$$

it is the unique number satisfying 


$$
f(\ell)=-\frac{1}{\ell}+\frac{1}{2 \ell} \int_{-\ell}^{\ell} f(x) d x
$$

We consider the initial value problem

$$
u_{t}+F\left(\left(\operatorname{sign} u_{x}\right)_{x}-f(x)\right)=0
$$

with $F(\xi)=-\xi$. By using above $\ell$ one gets an explicit solution.

Theorem 6.2 Assume that $f$ is as above. Let $\ell \in(0, R)$ be a unique number satisfying (6.2). Then the function

$$
u(x, t)=\max (-f(x) t,-f(\ell) t)
$$

is the unique solution of (6.3) with initial data $u_{0} \equiv 0$.

Since the facet is $\chi_{\ell}$ for all $t>0$, it is easy to see that this is a solution. For more examples of solutions see [13].

Acknowledgements The authors thank the anonymous referees for their careful reading of the manuscript and their comments. The first author was partly supported by the Japan Society for the Promotion of Sciences (JSPS) through Grant-in-Aid for Scientific Research Kiban A (No. 19H00639), Kiban A (No. 17H01091) and Challenging Pioneering Research (Kaitaku)(No. 18H05323). The second author was supported by JSPS KAKENHI Wakate Grant (No. 18K13440).

Open Access This article is licensed under a Creative Commons Attribution 4.0 International License, which permits use, sharing, adaptation, distribution and reproduction in any medium or format, as long as you give appropriate credit to the original author(s) and the source, provide a link to the Creative Commons licence, and indicate if changes were made. The images or other third party material in this article are included in the article's Creative Commons licence, unless indicated otherwise in a credit line to the material. If material is not included in the article's Creative Commons licence and your intended use is not permitted by statutory regulation or exceeds the permitted use, you will need to obtain permission directly from the copyright holder. To view a copy of this licence, visit http://creativecommons.org/licenses/by/4.0/.

\section{Appendix A: Regularity of the solution of the resolvent problem}

In the perturbed test function method in the proof of Theorem 4.1, we need to solve the resolvent problem for $\mathcal{E}_{f}$ defined in (1.4). Here we show that the solution of this resolvent problem is Lipschitz continuous. Suppose that $\psi$ is a Lipschitz function with Lipschitz constant $L$ and $f$ is a Lipschitz function with Lipschitz constant $L_{f}$. Consider the solution $\zeta$ of

$$
\zeta+a \partial \mathcal{E}_{f}(\zeta) \ni \psi
$$

By shifting $\zeta_{y}:=\zeta(\cdot-y)$, etc., we have

$$
\zeta_{y}+a \partial \mathcal{E}_{f_{y}}\left(\zeta_{y}\right) \ni \psi_{y}
$$

or, equivalently,

$$
\zeta_{y}+a \partial \mathcal{E}_{0}\left(\zeta_{y}\right) \ni \psi_{y}-a f_{y} .
$$

By the Lipschitz continuity, we have 


$$
\psi_{y}-a f_{y}-\left(L+a L_{f}\right)|y| \leq \psi-a f \leq \psi_{y}-a f_{y}+\left(L+a L_{f}\right)|y|
$$

and hence the comparison principle for the elliptic resolvent problem yields

$$
\zeta_{y}-\left(L+a L_{f}\right)|y| \leq \zeta \leq \zeta_{y}+\left(L+a L_{f}\right)|y| .
$$

We conclude that $\zeta$ is Lipschitz continuous with the Lipschitz constant $L+a L_{f}$.

\section{References}

1. Angenent, S., Gurtin, M.E.: Multiphase thermomechanics with interfacial structure. II. Evolution of an isothermal interface. Arch. Ration. Mech. Anal. 108(4), 323-391 (1989)

2. Anzellotti, G.: Pairings between measures and bounded functions and compensated compactness. Ann. Mat. Pura Appl. (4) 135, 293-318 (1983). https://doi.org/10.1007/BF01781073

3. Arisawa, M., Giga, Y.: Anisotropic curvature flow in a very thin domain. Indiana Univ. Math. J. 52(2), 257-281 (2003). https://doi.org/10.1512/iumj.2003.52.2099

4. Barles, G.: A weak Bernstein method for fully nonlinear elliptic equations. Differ. Integral Equ. 4(2), 241-262 (1991)

5. Bellettini, G., Goglione, R., Novaga, M.: Approximation to driven motion by crystalline curvature in two dimensions. Adv. Math. Sci. Appl. 10(1), 467-493 (2000). arXiv:1707.03342

6. Braides, A., Malusa, A., Novaga, M.: Crystalline evolutions with rapidly oscillating forcing terms (2017). arXiv:1707.03342

7. Crandall, M.G., Ishii, H., Lions, P.-L.: User's guide to viscosity solutions of second order partial differential equations. Bull. Am. Math. Soc. (N.S.) 27(1), 1-67 (1992). https://doi.org/10.1090/S02730979-1992-00266-5

8. Chambolle, A., Morini, M., Novaga, M., Ponsiglione, M.: Existence and uniqueness for anisotropic and crystalline mean curvature flows. J. Am. Math. Soc. 32(3), 779-824 (2019). https://doi.org/10.1090/ jams/919

9. Chambolle, A., Morini, M., Ponsiglione, M.: Existence and uniqueness for a crystalline mean curvature flow. Commun. Pure Appl. Math. 70(6), 1084-1114 (2017). https://doi.org/10.1002/cpa.21668

10. Chambolle, A., Novaga, M.: Existence and uniqueness for planar anisotropic and crystalline curvature flow, Variational methods for evolving objects. In: Giga, Y., Tonegawa, Y. (eds.) Adv. Stud. Pure Math., vol. 67, pp. 87-113, Math. Soc. Japan (2015). https://doi.org/10.2969/aspm/06710087

11. De Zan, C., Soravia, P.: A comparison principle for the mean curvature flow equation with discontinuous coefficients. Int. J. Differ. Equ. posted on 2016, Art. ID 3627896, 6 (2016) https://doi.org/10. $1155 / 2016 / 3627896$

12. Giga, Y.: Surface evolution equations: a level set approach. In: Monographs in Mathematics, vol. 99. Birkhauser, Basel (2006)

13. Giga, M.-H., Giga, Y.: A subdifferential interpretation of crystalline motion under nonuniform driving force. In: Dynamical Systems and Differential Equations, vol. I, pp. 276-287. Springfield (1998)

14. Giga, M.-H., Giga, Y.: Evolving graphs by singular weighted curvature. Arch. Ration. Mech. Anal. 141(2), 117-198 (1998)

15. Giga, M.-H., Giga, Y., Nakayasu, A.: On general existence results for one-dimensional singular diffusion equations with spatially inhomogeneous driving force. In: Geometric Partial Differential Equations, CRM Series, vol. 15, pp. 145-170. Ed. Norm., Pisa (2013)

16. Giga, M.-H., Giga, Y., Rybka, P.: A comparison principle for singular diffusion equations with spatially inhomogeneous driving force for graphs. Arch. Ration. Mech. Anal. 211(2), 419-453 (2014). https://doi. org/10.1007/s00205-013-0676-y. (Erratum: 212(2014), 707)

17. Giga, Y., Gorka, P., Rybka, P.: Nonlocal spatially inhomogeneous Hamilton-Jacobi equation with unusual free boundary. Discrete Contin. Dyn. Syst. 26(2), 493-519 (2010). https://doi.org/10.3934/dcds. 2010.26.493

18. Giga, Y., Gorka, P., Rybka, P.: A comparison principle for Hamilton-Jacobi equations with discontinuous Hamiltonians. Proc. Am. Math. Soc. 139(5), 1777-1785 (2011). https://doi.org/10.1090/S00029939-2010-10630-5

19. Giga, Y., Gorka, P., Rybka, P.: Evolution of regular bent rectangles by the driven crystalline curvature flow in the plane with a non-uniform forcing term. Adv. Differ. Equ. 18(3-4), 201-242 (2013) 
20. Giga, Y., Ohnuma, M., Sato, M.-H.: On the strong maximum principle and the large time behavior of generalized mean curvature flow with the Neumann boundary condition. J. Differ. Equ. 154(1), 107-131 (1999). https://doi.org/10.1006/jdeq.1998.3569

21. Giga, Y., Pozar, N.: A level set crystalline mean curvature flow of surfaces. Adv. Differ. Equ. 21(7-8), 631-698 (2016)

22. Giga, Y., Pozar, N.: Approximation of general facets by regular facets with respect to anisotropic total variation energies and its application to crystalline mean curvature flow. Commun. Pure Appl. Math. 71(7), 1461-1491 (2018). https://doi.org/10.1002/cpa.21752

23. Giga, Y., Rybka, P.: Stability of facets of self-similar motion of a crystal. Adv. Differ. Equ. 10(6), 601-634 (2005)

24. Giga, Y., Rybka, P.: Stability of facets of crystals growing from vapor. Discrete Contin. Dyn. Syst. 14(4), 689-706 (2006). https://doi.org/10.3934/dcds.2006.14.689

25. Giga, Y., Rybka, P.: Facet bending in the driven crystalline curvature flow in the plane. J. Geom. Anal. 18(1), 109-147 (2008). https://doi.org/10.1007/s12220-007-9004-9

26. Giga, Y., Rybka, P.: Faceted crystal grown from solution-a Stefan type problem with a singular interfacial energy. In: Fujita, H., Nakamura, M. (eds.) Proceedings of the 4th JSAM-SIMAI Seminar on Industrial and Applied Mathematics, Gakuto International Series, Mathematical Sciences and Applications 28, Gakkotosho, Tokyo, pp. 31-41 (2008)

27. Giga, Y., Rybka, P.: Facet bending driven by the planar crystalline curvature with a generic nonuniform forcing term. J. Differ. Equ. 246(6), 2264-2303 (2009). https://doi.org/10.1016/j.jde.2009.01.009

28. Ladyzenskaja, O.A., Solonnikov, V.A., Ural'ceva, N.N.: Linear and quasilinear equations of parabolic type, Translated from the Russian by S. Smith. Translations of Mathematical Monographs, vol. 23. American Mathematical Society, Providence (Russian) (1968)

29. Lieberman, G.M.: Second Order Parabolic Differential Equations. World Scientific Publishing Co., Inc, River Edge (1996)

30. Lunardi, A.: Analytic semigroups and optimal regularity in parabolic problems. In: Modern Birkhauser Classics. Birkhauser/Springer Basel AG, Basel [2013 reprint of the 1995 original] (1995)

31. Mucha, P.B., Rybka, P.: A new look at equilibria in Stefan-type problems in the plane. SIAM J. Math. Anal. 39(4), 1120-1134 (2007/2008). https://doi.org/10.1137/060677124

32. Mucha, P.B., Rybka, P.: A caricature of a singular curvature flow in the plane. Nonlinearity 21(10), 2281-2316 (2008). https://doi.org/10.1088/0951-7715/21/10/005

33. Taylor, J.E.: Constructions and conjectures in crystalline nondifferential geometry. In: Lawson, B., Tanenblat, K. (eds.) Differential geometry, Proceedings of the Conference on Differential Geometry, Rio de Janeiro, Pitman Monogr. Surveys Pure Appl. Math., vol. 52, pp. 321-336. Longman Sci. Tech., Harlow (1991). https://doi.org/10.1111/J.1439-0388.1991.tb00191.x 